\title{
TRADING NETWORKS WITH FRICTIONS
}

\section{TAMÁS FLEINER}

Department of Computer Science and Information Theory, Budapest University of Technology and Economics and Institute of Economics, Centre for Economic and Regional Studies,

Hungarian Academy of Sciences

\section{RAVI JAGADEESAN}

Harvard Business School and Department of Economics, Harvard University

\author{
ZSUZSANNA JANKÓ \\ Department of Mathematics, University of Hamburg and Department of Operations Research and Actuarial \\ Sciences, Corvinus University of Budapest \\ AleXANDER TEYTELBOYM \\ Department of Economics, Institute for New Economic Thinking at the Oxford Martin School, and \\ St. Catherine's College, University of Oxford
}

\begin{abstract}
We show how frictions and continuous transfers jointly affect equilibria in a model of matching in trading networks. Our model incorporates distortionary frictions such as transaction taxes and commissions. When contracts are fully substitutable for firms, competitive equilibria exist and coincide with outcomes that satisfy a cooperative solution concept called trail stability. However, competitive equilibria are generally neither stable nor Pareto-efficient.
\end{abstract}

KEYwORDS: Trading networks, frictions, competitive equilibrium, matching with contracts, trail stability, transaction taxes, commissions.

\section{INTRODUCTION}

INTERDEPENDENCE AND SPECIALIZATION OF PRODUCTION are central features of the modern economy. Many firms have complex, bilateral relationships with dozens of buy-

\footnotetext{
Tamás Fleiner: fleiner@cs.bme.hu

Ravi Jagadeesan: ravi.jagadeesan@gmail.com

Zsuzsanna Jankó: zsuzsanna.janko@uni-hamburg.de

Alexander Teytelboym: alexander.teytelboym@economics.ox.ac.uk

This paper combines and supersedes parts of the previous working papers "Trading networks with bilateral contracts" by Tamás Fleiner, Zsuzsanna Jankó, Akihisa Tamura, and Alexander Teytelboym and "Matching without transfers is general equilibrium with incomplete markets" by Ravi Jagadeesan. An extended abstract of this paper appeared in the Proceedings of the 2018 ACM Conference on Economics and Computation (EC'18). We would like to thank Samson Alva, David Delacrétaz, Battal Doğan, Laura Doval, Jeremy Fox, Daniel Gottlieb, Jens Gudmundsson, Onur Kesten, Maia King, Bettina Klaus, Scott Kominers, Shengwu Li, Michael Ostrovsky, Madhav Raghavan, Larry Samuelson, Jan Christoph Schlegel, Steven Shadman, Fabien Wang, the anonymous referees, and seminar participants at CMU/University of Pittsburgh, ETH, Harvard, Oxford, and the NBER Market Design Working Group Meeting for their valuable comments on this paper. Fleiner was supported by a Cooperation of Excellences Grant (KEP-6/2018), the OTKA K108383 and K128611 research projects, and the MTA-ELTE Egerváry Research Group. Part of this research was conducted while Jagadeesan was an Economic Design Fellow at the Harvard Center of Mathematical Sciences and Applications. Jagadeesan gratefully acknowledges the support of a National Science Foundation Graduate Research Fellowship under Grant DGE-1745303. Jankó was supported by a Cooperation of Excellences Grant (KEP-6/2018), OTKA Grants K109240 and K128611, and the MTA-ELTE Egerváry Research Group. Teytelboym was supported by Economic and Social Research Council Grant ES/R007470/1. The research reported in this paper has been supported by the National Research, Development and Innovation Fund (TUDFO/51757/2019-ITM, Thematic Excellence Program).
} 
ers and suppliers. The terms of these relationships are typically encoded in discrete contracts that specify goods traded or services rendered, delivery dates, penalties for noncompletion, and, of course, prices. Models of matching with contracts, inspired by Gale and Shapley (1962), elegantly capture discrete interactions in highly differentiated markets with heterogeneous agents (Crawford and Knoer (1981), Kelso and Crawford (1982), Roth (1984), Hatfield and Milgrom (2005)). We focus on matching in trading networks in order to represent complex production linkages in the economy.

Trading networks can suffer from distortionary frictions, such as transaction taxes and broker commissions. Distortionary frictions introduce wedges between payments and receipts and therefore make utility imperfectly transferable between agents. Despite the practical relevance of distortionary frictions, most previous models of matching in trading networks with continuous prices assume that utility is perfectly transferable between agents (Hatfield, Kominers, Nichifor, Ostrovsky, and Westkamp (2013)).

In this paper, we develop a theory of trading networks in which there are continuous transfers between agents but distortionary frictions make utility imperfectly transferable. We establish two results. First, we provide sufficient conditions for the existence of competitive equilibria that apply in the presence of discrete contracts and distortionary frictions. Second, we show that there is an equivalence between competitive equilibrium and an intuitive cooperative solution concept. Our equivalence result is in the spirit of $\mathrm{Au}$ mann (1964) but holds for fixed, finite markets and applies in the presence of frictions. Therefore, as we will argue, our equivalence result provides new cooperative foundations for competitive equilibrium and competitive foundations for the cooperative solution concept that we consider.

To model trading networks, we follow Hatfield et al. (2013) and Hatfield, Kominers, Nichifor, Ostrovsky, and Westkamp (2018) and assume that agents interact via an exogenously specified set of bilateral trades. A trade specifies who is trading, what good or service is being traded, and any non-pecuniary parameters of exchange. Trades also have directions that correspond to the flow of goods: upstream trades represent purchases and downstream trades represent sales (Ostrovsky (2008)). In a market outcome, transfers are made for every realized trade, encapsulating the role of money in the economy. An outcome is summarized by a set of realized contracts, each of which specifies a trade and a price.

To model distortionary frictions, we introduce a novel ingredient to the trading network framework. Specifically, we allow agents to place different values on transfers associated to different trades in order to capture wedges between payments and receipts. This feature allows our model to capture frictions such as transaction taxes and commissions.

Our first main result provides sufficient conditions for the existence of competitive equilibria. The key condition for existence is that preferences over contracts are fully substitutable (Ostrovsky (2008), Hatfield and Kominers (2012), Hatfield et al. (2013)) - that is, that upstream (resp. downstream) trades are grossly substitutable for each other, and that upstream and downstream trades are grossly complementary to one another. Full substitutability can be regarded as the requirement that the goods that flow in trades are grossly substitutable (Baldwin and Klemperer (2019), Hatfield, Kominers, Nichifor, Ostrovsky, and Westkamp (2019)). We show that full substitutability and a mild regularity condition together ensure that competitive equilibria exist in our model. ${ }^{1}$

\footnotetext{
${ }^{1}$ As Hatfield and Kominers (2012) (resp. Hatfield et al. (2013)) showed, full substitutability is necessary in a maximal domain sense for the existence of equilibria in trading networks with discrete and bounded prices (resp. with transferable utility).
} 
To relate the competitive and cooperative approaches to the analysis of markets with frictions, we first explore cooperative interpretations of competitive equilibria. Standard solution concepts in matching theory are stability (in the sense of Hatfield et al. (2013)) which requires that there is no group of firms that can commit to recontracting among themselves (while possibly dropping some existing contracts) - and the core. However, we show that in the presence of frictions, competitive equilibrium outcomes are generally neither stable nor in the core. Moreover, stable outcomes do not exist in general even when contracts are fully substitutable.

Therefore, we use a different cooperative solution concept to analyze trading networks with frictions. An outcome is trail-stable if it is immune to sequential deviations in which a firm that receives an upstream (resp. downstream) contract offer can either accept the offer outright or make an additional downstream (resp. upstream) contract offer (Fleiner, Jankó, Tamura, and Teytelboym (2018)). Trail stability is an extension of Gale and Shapley's (1962) pairwise stability property to trading networks. We show that competitive equilibrium outcomes are always trail-stable. In particular, trail-stable outcomes exist even in the presence of frictions whenever competitive equilibria exist.

Trail stability also has a competitive interpretation. We show that trail-stable outcomes can be supported by competitive equilibrium prices under full substitutability and regularity conditions. As an outcome is a set of realized contracts, it only specifies the prices of realized trades. Hence, the crucial part of the proof is to construct equilibrium prices for unrealized trades as well. Our results show that trail-stable outcomes are essentially equivalent to competitive equilibria-even in the presence of frictions.

From an applied perspective, our model may be of interest to structural econometricians. Recent work on estimation in matching markets with transfers has focused on frictionless trading networks (Fox (2017, 2018), Fox, Hsu, and Yang (2018)) and two-sided markets with frictions (Cherchye, Demuynck, De Rock, and Vermeulen (2017), Galichon, Kominers, and Weber (2019)). Our model allows for both frictions and interconnectedness, which are both key features of markets such as the real estate market. Structural methods based on our model would allow an econometrician to partially identify preferences by assuming that the observed market outcome is trail-stable-or, equivalently, obtained from a competitive equilibrium.

Most previous models of matching in trading networks imposed significant additional conditions on the structure of the trading network, the space of contracts, or preferences. Ostrovsky (2008), Westkamp (2010), and Hatfield and Kominers (2012) derived existence and structural results for acyclic networks, which cannot contain "horizontal" trade between intermediaries. Hatfield et al. (2018), Fleiner et al. (2018), and Adachi (2017) extended the analysis of Ostrovsky (2008) to general trading networks. However, Ostrovsky (2008), Westkamp (2010), Hatfield and Kominers (2012), and Fleiner et al. (2018) all assumed that there are finitely many contracts-ruling out continuous or unbounded prices and precluding comparisons with competitive equilibrium. Hatfield et al. (2013) considered general trading networks with continuous prices and technological constraints, but assumed that utility is perfectly transferable-ruling out both distortionary frictions and income effects. ${ }^{2}$ Hatfield et al. (2018) introduced continuous prices into discrete models of matching in trading networks-allowing for technological constraints and without assuming that utility is transferable. Our model specializes that of Hatfield et al. (2018) to accommodate an analysis of competitive equilibria. Hatfield et al. (2018) also analyzed

\footnotetext{
${ }^{2}$ Hatfield et al. (2013) allowed for fixed transaction costs, such as shipping costs and lump-sum transaction taxes, but not for proportional transaction taxes and commissions.
} 
the properties of stable outcomes-which do not generally exist in our model even when competitive equilibria exist. In contrast, we focus on the existence of trail-stable outcomes and their relationship to competitive equilibria.

Our work also builds on large literatures on one-to-one and many-to-one matching with imperfectly transferable utility (see, e.g., Dupuy, Galichon, Jaffe, and Kominers (2017) and Galichon et al. (2019)). The source of imperfect transferability-that is, whether it is due to frictions or income effects-is irrelevant in both one-to-one and many-to-one matching. In our model, on the other hand, frictions can cause trail-stable outcomes and competitive equilibria to be Pareto-inefficient, while income effects do not give rise to Pareto inefficiency.

More broadly, our paper builds on a rich literature on competitive equilibrium with indivisible goods. In that literature, it is typically assumed that utility is perfectly transferable (see, e.g., Gul and Stacchetti (1999), Sun and Yang (2006), and Baldwin and Klemperer (2019)), ruling out both distortionary frictions and income effects. Danilov, Koshevoy, and Murota (2001) showed that competitive equilibria exist for certain classes of preferences with complementarities and income effects, but they ruled out frictions. We assume a substitutability condition, but allow for both frictions and income effects.

This paper proceeds as follows. Section 2 introduces the model. Section 3 explains how our model captures frictions and describes leading examples. Section 4 presents sufficient conditions for the existence of competitive equilibria. Section 5 defines trail stability and relates it to competitive equilibrium. Section 6 describes how competitive equilibrium and trail stability relate to other cooperative solution concepts. (We present the details of these relationships in Appendix E in the Supplemental Material (Fleiner, Jagadeesan, Jankó, and Teytelboym (2019)).) Section 7 is a conclusion. Appendix A formulates equivalent definitions of full substitutability that we use in the proofs of our main results. (We prove that the definitions are equivalent in Appendix D in the Supplemental Material.) Appendices $\mathrm{B}$ and $\mathrm{C}$ contain the proofs of the results in the text.

\section{MODEL}

Our model is based on that of Hatfield et al. (2018) but requires that prices be continuous and unbounded.

\subsection{Firms and Contracts}

There is a finite set $F$ of firms and a finite set $\Omega$ of trades. Each trade $\omega \in \Omega$ is associated to a buyer $\mathrm{b}(\omega) \in F$ and a seller $\mathrm{s}(\omega) \in F$. Trades specify what is being exchanged as well as any non-pecuniary contract terms (Hatfield et al. (2013)).

A contract is a pair $\left(\omega, p_{\omega}\right)$ that consists of a trade $\omega$ and a price $p_{\omega} \in \mathbb{R}$ for $\omega$. Thus, the set of contracts is $X=\Omega \times \mathbb{R}$. For a set of contracts $Y \subseteq X$, we let

$$
\tau(Y)=\left\{\omega \in \Omega \mid\left(\omega, p_{\omega}\right) \in Y \text { for some } p_{\omega}\right\}
$$

denote the set of trades that are associated to contracts in $Y$. An outcome is a set $Y \subseteq X$ of contracts such that each trade is associated with at most one price in $Y$-formally, $|\tau(Y)|=|Y|$.

Given a set $\Xi \subseteq \Omega$ of trades and a firm $f \in F$, let $\Xi_{\rightarrow f}$ denote the set of trades in $\Xi$ in which $f$ acts as a buyer, let $\Xi_{f \rightarrow}$ denote the set of trades in $\Xi$ in which $f$ acts as a seller, and let $\Xi_{f}=\Xi_{\rightarrow f} \cup \Xi_{f \rightarrow}$ denote the set of trades in $\Xi$ in which $f$ is involved (as either 
a buyer or as a seller). For a set $Y \subseteq X$ of contracts, we define subsets $Y_{\rightarrow f}, Y_{f \rightarrow}$, and $Y_{f}$ analogously.

An arrangement is a pair $[\Xi ; p]$ of a set of trades $\Xi \subseteq \Omega$ and a price vector $p \in \mathbb{R}^{\Omega}$. Given an arrangement $[\Xi ; p]$, we define an associated outcome $\kappa([\Xi ; p]) \subseteq X$ by

$$
\kappa([\Xi ; p])=\left\{\left(\omega, p_{\omega}\right) \mid \omega \in \Xi\right\} .
$$

That is, $\kappa([\Xi ; p])$ is the outcome at which the trades in $\Xi$ are realized at the prices given by $p$. Unlike outcomes, arrangements specify prices even for unrealized trades.

\subsection{Utility Functions and Transfers}

Each firm's utility depends only on the trades that involve the firm and on the transfers that it receives. Formally, firm $f$ has a utility function $u^{f}: \mathcal{P}\left(\Omega_{f}\right) \times \mathbb{R}^{\Omega_{f}} \rightarrow \mathbb{R} \cup\left\{-\infty\right.$. $^{3}$

Three features of our specification of utility functions are worth highlighting. First, the utility function depends on the entire vector of transfers (as opposed to merely on net transfers). Hence, we allow firms to place different marginal values on transfers associated to different trades; as we show in Section 3, this feature allows our model to capture distortionary frictions. Second, we allow the utility function to take value $-\infty$ to capture technological constraints (Hatfield et al. (2013)). Specifically, we set $u^{f}(\Xi, t)=-\infty$ for all transfer vectors $t$ if the set $\Xi$ of trades is technologically infeasible for $f$. Last, while utility depends on the transfers associated to unrealized trades, firms do not receive transfers for unrealized trades in market outcomes.

We assume that $u^{f}(\Xi, t)$ is continuous in $t$ and that

$$
t \leq t^{\prime} \quad \Longrightarrow \quad u^{f}(\Xi, t) \leq u^{f}\left(\Xi, t^{\prime}\right)
$$

with equality if and only if $u^{f}(\Xi, t)=-\infty$, so transfers are relevant to firms whenever a set of trades is feasible. We also assume that $u^{f}(\emptyset, 0) \in \mathbb{R}$, so autarky is feasible. The transferable utility trading network model of Hatfield et al. (2013) is recovered when

$$
u^{f}(\Xi, t)=v^{f}(\Xi)+\sum_{\omega \in \Omega_{f}} t_{\omega}
$$

for some valuation function $v^{f}: \mathcal{P}\left(\Omega_{f}\right) \rightarrow \mathbb{R} \cup\{-\infty\}$.

To analyze competitive equilibria, we need to consider firms' demands for trades at any given price vector. Prices give rise to transfers in the following manner. Firms receive no transfer for a trade if they do not agree to the trade. Firms receive transfers equal to the prices of any realized sales (downstream trades) and pay transfers equal to the prices of any realized purchases (upstream trades). Maximizing utility at a price vector $p \in \mathbb{R}^{\Omega_{f}}$ gives rise to a collection of sets of demanded trades

$$
D^{f}(p)=\underset{\Xi \subseteq \Omega_{f}}{\arg \max } u^{f}\left(\Xi,\left(p_{\Xi_{f \rightarrow}},(-p)_{\Xi_{\rightarrow f}}, 0_{\Omega_{f} \backslash \Xi}\right)\right) .
$$

Thus, $D^{f}$ is the demand correspondence of firm $f$.

As is typical in matching theory, we also need to consider firms' choices from sets of available contracts. Given a firm $f$ and an outcome $Y \subseteq X_{f}$, the utility of $Y$ for $f$ is

\footnotetext{
${ }^{3}$ We write $\mathcal{P}(Z)$ for the power set of a set $Z$.
} 
$U^{f}(Y)=u^{f}\left(\tau(Y), t^{f}(Y)\right)$, where $t^{f}(Y)_{\omega}$ is the transfer associated with trade $\omega$ in $Y{ }^{4}$ We define the choice correspondence $C^{f}: \mathcal{P}\left(X_{f}\right) \rightrightarrows \mathcal{P}\left(X_{f}\right)$ by

$$
C^{f}(Y)=\underset{\text { outcomes } Z \subseteq Y}{\arg \max } U^{f}(Z) .
$$

Since prices are continuous, firms may be indifferent between certain outcomes, so the choice correspondence is generally multi-valued.

\subsection{Competitive Equilibrium}

In a competitive equilibrium, firms act as price-takers and all markets clear. Here, the market for a trade clears at a specified price if the trade is either demanded by both the buyer and the seller or it is demanded by neither. Therefore, in a competitive equilibrium, buyers and sellers demand the same sets of trades at the prices that they face. As in Hatfield et al. (2013), in order to fully specify a competitive equilibrium, we need to assign prices to all trades-including ones that are not realized.

DEFINITION 1: An arrangement $[\Xi ; p]$ is a competitive equilibrium if $\Xi_{f} \in D^{f}\left(p_{\Omega_{f}}\right)$ for all $f$.

As interchangeable trades with different counterparties can be priced differently, our competitive equilibria can have personalized prices (as in Hatfield et al. (2013)). ${ }^{5}$ We call an outcome $A$ a competitive equilibrium outcome if $A=\kappa([\Xi ; p])$ for some competitive equilibrium $[\Xi ; p]$.

\section{DISTORTIONARY FRICTIONS}

In our model, firms may value transfers from different trades differently, so a unit of $t_{\omega}$ might be worth less to the firm than a unit of $t_{\omega^{\prime}}{ }^{6}$ This feature allows our model to capture (in a reduced form) distortionary frictions such as variable transaction taxes and commissions. This section illustrates exactly how our model can capture transaction taxes and how they can in turn affect competitive equilibria.

\subsection{Capturing Transaction Taxes}

Suppose, for example, that $\lambda$ proportion of every transfer must be paid to the government as a tax. We assume that the recipient of the transfer pays the transaction tax-this

${ }^{4}$ Formally, we define a vector $t^{f}(Y) \in \mathbb{R}^{\Omega_{f}}$ by letting

$$
t^{f}(Y)_{\omega}= \begin{cases}0 & \text { if } \omega \notin \tau(Y), \\ p_{\omega} & \text { if }\left(\omega, p_{\omega}\right) \in Y_{f \rightarrow}, \\ -p_{\omega} & \text { if }\left(\omega, p_{\omega}\right) \in Y_{\rightarrow f}\end{cases}
$$

for each trade $\omega \in \Omega_{f}$.

${ }^{5}$ For example, trades of the same good with different counterparties can have different prices in a competitive equilibrium.

${ }^{6}$ That is, firms can have different marginal rates of substitution between transfers associated to different trades. 
assumption is without loss of generality. Thus, the net-of-tax transfer received or paid by a firm for a trade $\omega$ is

$$
T_{\omega}\left(t_{\omega}\right)= \begin{cases}(1-\lambda) t_{\omega} & \text { if } t_{\omega} \geq 0, \\ t_{\omega} & \text { if } t_{\omega}<0,\end{cases}
$$

where $t_{\omega}$ is the gross transfer (Dupuy et al. (2017)). Hence, when $t_{\omega} \geq 0$, the firm is a recipient of the transfer and receives $(1-\lambda) t_{\omega}$; when $t_{\omega}<0$, the firm is a payer and pays $\left|t_{\omega}\right|$ in full. As a result, if firm $f$ has quasilinear preferences and valuation function $v^{f}: \mathcal{P}\left(\Omega_{f}\right) \rightarrow \mathbb{R} \cup\{-\infty\}$, then the utility function $u^{f}$ is

$$
u^{f}(\Xi, t)=v^{f}(\Xi)+\sum_{\omega \in \Omega_{f}} T_{\omega}\left(t_{\omega}\right) .
$$

When $\lambda<1$ and $v^{f}(\emptyset) \in \mathbb{R}$, the utility function $u^{f}$ satisfies our conditions on preferences (i.e., it is continuous and satisfies the requisite monotonicity condition). Note that transaction taxes make utility imperfectly transferable even if preferences are quasilinear. ${ }^{7}$

We can model transaction taxes similarly even in the presence of income effects. If firm $f$ has utility function $\widehat{u}^{f}$ before taxes, then the net-of-tax utility function is

$$
u^{f}(\Xi, t)=\widehat{u}^{f}\left(\Xi,\left(T_{\omega}\left(t_{\omega}\right)\right)_{\omega \in \Omega_{f}}\right) .
$$

More generally, our framework can capture nonlinear transaction taxes and subsidies. If a tax of $\Lambda_{\omega}\left(t_{\omega}\right)$ must be paid on a transfer of size $t_{\omega} \geq 0$ for trade $\omega$, then we can take the net-of-tax transfer function $T_{\omega}$ to be

$$
T_{\omega}\left(t_{\omega}\right)= \begin{cases}t_{\omega}-\Lambda_{\omega}\left(t_{\omega}\right) & \text { if } t_{\omega} \geq 0, \\ t_{\omega} & \text { if } t_{\omega}<0,\end{cases}
$$

in (4) to define the net-of-tax utility function. The case of $\Lambda_{\omega}\left(t_{\omega}\right)=\lambda t_{\omega}$ recovers the proportional transaction tax discussed above. If $\widehat{u}^{f}$ is continuous and satisfies the requisite monotonicity condition and marginal tax rates are strictly less than $1,{ }^{8}$ then $u^{f}$ is continuous and satisfies the requisite monotonicity condition as well. It is straightforward to extend the definition of $T_{\omega}$ to capture direction-dependent transaction taxes.

Commissions that comprise a fraction of sale prices (such as real estate commissions) can be straightforwardly represented as transaction taxes. More generally, as our framework allows for imperfectly transferable utility, it can capture settings in which there is a complex set of feasible surplus distributions between counterparties (Galichon et al. (2019)). ${ }^{9}$ For sake of simplicity, we focus on transaction taxes as the source of imperfect transferability in our leading examples.

\subsection{Leading Examples}

We now illustrate how distortionary frictions can affect competitive equilibria. The first example considers a cyclic economy in which firms have quasilinear preferences and trans-

\footnotetext{
${ }^{7}$ We thank a referee for this observation.

${ }^{8}$ Formally, we require that $\Lambda_{\omega}$ be continuous, that $\Lambda_{\omega}(0)=0$, and that $x_{2}-\Lambda_{\omega}\left(x_{2}\right)<x_{1}-\Lambda_{\omega}\left(x_{1}\right)$ for all $x_{1}>x_{2}>0$ to ensure that the utility functions satisfy the requisite monotonicity condition.

${ }^{9}$ For example, as Galichon et al. (2019) showed, matching models with imperfectly transferable utility can capture the "collective" model of intra-household bargaining (Chiappori (1988)).
} 


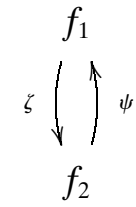

(a) Example 1.

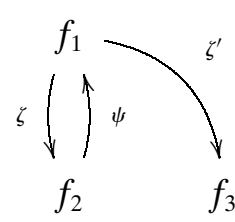

(b) Example 2 .

FIGURE 1.-Trades in Examples 1 and 2. Arrows point from sellers to buyers.

action taxes are incorporated as discussed in Section 3.1. We show that competitive equilibria can be Pareto-inefficient and hence that competitive equilibrium outcomes are generally not in the core.

EXAMPLE 1-A Pareto-Inefficient Competitive Equilibrium: As depicted in Figure 1(a), there are two firms, $f_{1}$ and $f_{2}$, which interact via two trades. There is a proportional transaction tax of $\lambda=10 \%$. The firms share the same quasilinear utility function (see (2) and (3)) with valuation $v=v^{f_{1}}=v^{f_{2}}$ defined by

$$
\begin{aligned}
v(\emptyset) & =0, \\
v(\{\zeta, \psi\}) & =10, \\
v(\{\zeta\})=v(\{\psi\}) & =-\infty .
\end{aligned}
$$

There are two sets of trades that can be supported in competitive equilibria: namely, $\emptyset$ and $\{\zeta, \psi\}$. For example, the arrangement $[\{\zeta, \psi\} ; p]$ is a competitive equilibrium if $-100 \leq p_{\zeta}=p_{\psi} \leq 100$, and the arrangement $[\varnothing ; p]$ is a competitive equilibrium if $p_{\zeta}=p_{\psi} \geq 100$ or $p_{\zeta}=p_{\psi} \leq-100 .{ }^{10}$ The competitive equilibria that support the autarky outcome are all Pareto-inefficient. ${ }^{11}$

In contrast, in markets without transaction taxes or other distortionary frictions, competitive equilibria are in the core (and hence Pareto-efficient).

The second example shows that adding an outside option for $f_{1}$ to Example 1 can shut down trade between $f_{1}$ and $f_{2}$ due to pecuniary externalities. In the context of Examples 1 and 2, adding an outside option can cause prices to become extreme, inducing heavy trading losses (due to taxes) that shut down the market.

EXAMPLE 2-An Outside Option That Shuts Down a Market: As depicted in Figure $1(\mathrm{~b})$, there are three firms, $f_{1}, f_{2}$, and $f_{3}$, which interact via three trades. There is a proportional transaction tax of $\lambda=10 \%$. The firms have quasilinear utility functions

\footnotetext{
${ }^{10}$ In general, the arrangement $[\{\zeta, \psi\} ; p]$ is a competitive equilibrium if and only if

$$
\min \left\{p_{\zeta}, 0.9 p_{\zeta}\right\}+\min \left\{-p_{\psi},-0.9 p_{\psi}\right\} \geq-10 \quad \text { and } \min \left\{-p_{\zeta},-0.9 p_{\zeta}\right\}+\min \left\{p_{\psi}, 0.9 p_{\psi}\right\} \geq-10 .
$$
}

Similarly, the arrangement $[\emptyset ; p]$ is a competitive equilibrium if and only if

$$
\min \left\{p_{\zeta}, 0.9 p_{\zeta}\right\}+\min \left\{-p_{\psi},-0.9 p_{\psi}\right\} \leq-10 \quad \text { and } \min \left\{-p_{\zeta},-0.9 p_{\zeta}\right\}+\min \left\{p_{\psi}, 0.9 p_{\psi}\right\} \leq-10 .
$$

\footnotetext{
${ }^{11}$ The Pareto inefficiency in Example 1 persists even if tax revenue is remitted back to firms in the market, as $f_{1}$ and $f_{2}$ fail to realize any of the potential gains from trade at the autarky outcome.
} 
(see (2) and (3)), where we let $v^{f_{i}}$ denote the valuation of firm $f_{i}$. We let $v^{f_{i}}(\emptyset)=0$ for all firms. Extending Example 1, firm $f_{1}$ 's valuation is defined by

$$
\begin{gathered}
v^{f_{1}}(\{\zeta, \psi\})=v^{f_{1}}\left(\left\{\zeta^{\prime}, \psi\right\}\right)=10, \\
v^{f_{1}}(\{\zeta\})=v^{f_{1}}\left(\left\{\zeta^{\prime}\right\}\right)=v^{f_{1}}(\{\psi\})=-\infty, \\
v^{f_{1}}\left(\left\{\zeta, \zeta^{\prime}\right\}\right)=v^{f_{1}}\left(\left\{\zeta, \zeta^{\prime}, \psi\right\}\right)=-\infty .
\end{gathered}
$$

As in Example 1, firm $f_{2}$ 's valuation is defined by

$$
\begin{aligned}
v^{f_{2}}(\{\zeta, \psi\}) & =10, \\
v^{f_{2}}(\{\zeta\})=v^{f_{2}}(\{\psi\}) & =-\infty .
\end{aligned}
$$

Firm $f_{3}$ 's valuation is defined by $v^{f_{3}}\left(\left\{\zeta^{\prime}\right\}\right)=300$.

Trade $\zeta^{\prime}$ cannot be realized in equilibrium due to the technological constraints of $f_{1}$ and $f_{2} \cdot{ }^{12}$ Hence, we must have that $p_{\zeta^{\prime}} \geq 300$ in every competitive equilibrium, as $f_{3}$ must weakly prefer $\emptyset$ over $\left\{\zeta^{\prime}\right\}$ in equilibrium. For trade to occur, $f_{1}$ would have to prefer $\zeta$ over $\zeta^{\prime}$, and so we would have that $p_{\zeta} \geq p_{\zeta^{\prime}} \geq 300$. With $10 \%$ taxation and $p_{\zeta} \geq 300$, at least $\$ 30$ in taxes would have to be paid if $\zeta$ were realized. But $\$ 30$ exceeds the gains from trade between $f_{1}$ and $f_{2}$, so trade cannot occur in any competitive equilibrium despite the presence of gains from trade. An example of a competitive equilibrium is $[\emptyset ; p]$, where $p_{\zeta}=p_{\psi}=p_{\zeta^{\prime}}=350$. Thus, introducing an outside option that is not used in equilibrium can shut down a market when there are distortionary frictions.

In contrast, without transaction taxes, adding an outside option cannot cause competitive equilibria to become Pareto-inefficient (by the First Welfare Theorem).

\section{EXISTENCE OF COMPETITIVE EQUILIBRIA}

Because trades are indivisible, competitive equilibria need not exist in our model without further assumptions on preferences. The key condition for our existence result is full substitutability (Hatfield et al. (2013)). ${ }^{13}$

Intuitively, full substitutability requires that every firm view its upstream trades as gross substitutes for each other, its downstream trades as gross substitutes for each other, and its upstream and downstream trades as gross complements for one another.$^{14}$ More precisely, full substitutability requires that expansions in the set of upstream (resp. downstream) options and contractions in the set of downstream (resp. upstream) options only make upstream (resp. downstream) contracts less attractive and downstream (resp. upstream) contracts more attractive for a firm.

\footnotetext{
${ }^{12}$ To see why, note that if $\zeta^{\prime}$ is realized, then $\psi$ must be realized and $\zeta$ cannot be realized (due to $f_{1}$ 's preferences). But $f_{2}$ 's preferences require that $\zeta$ be realized whenever $\psi$ is realized.

${ }^{13}$ Full substitutability generalizes gross substitutability (Kelso and Crawford (1982), Gul and Stacchetti (1999)). We use the choice-language full substitutability condition introduced by Hatfield et al. (2013), which extends the same-side substitutability and cross-side complementarity conditions of Ostrovsky (2008) to choice correspondences.

${ }^{14}$ Section IIB in Hatfield et al. (2013) provides a detailed discussion of the full substitutability condition in the context of trading networks with transferable utility. For example, full substitutability rules out complementarities between inputs.
} 
AssUMPTION 1-Full Substitutability (FS)—Hatfield et al. (2013): For all $f \in F$ and all finite sets of contracts $Y, Y^{\prime} \subseteq X_{f}$ with $Y_{f \rightarrow \subseteq} \subseteq Y_{f \rightarrow}^{\prime}$ and $Y_{\rightarrow f} \supseteq Y_{\rightarrow f}^{\prime}$, if $C^{f}(Y)=\{Z\}$ and $C^{f}\left(Y^{\prime}\right)=\left\{Z^{\prime}\right\}$, then we have that $Z^{\prime} \cap Y_{f \rightarrow \subseteq Z}$ and that $Z \cap Y_{\rightarrow f}^{\prime} \subseteq Z^{\prime}$.

Technically, we impose the full substitutability condition only on sets of contracts from which a firm's utility-maximizing choice is unique. In Appendix A, we show that full substitutability is equivalent to a substitutability property that deals with indifferences more explicitly and to the weak quasisubmodularity of the indirect utility function (in a sense similar to Hatfield, Jagadeesan, and Kominers (2019)). ${ }^{15}$

Hatfield et al. (2013) also needed to assume that firms' valuations of sets of trades are never $+\infty$ to ensure that competitive equilibria exist. We impose a similar condition that is adapted to settings in which utility is not perfectly transferable. Our condition requires that the compensating variations of moving from autarky to trade be bounded belowthat is, that no set of trades be so desirable that it is preferred to autarky at any level of net transfers. This condition is satisfied in transferable utility economies when valuations are bounded above.

AssumPTION 2-Bounded Compensating Variations (BCV): For all $f \in F$, we have

$$
\inf _{(\Xi, t) \mid u^{f}(\Xi, t) \geq u^{f}(\emptyset, 0)} \sum_{\omega \in \Omega_{f}} t_{\omega}>-\infty .
$$

$\mathrm{BCV}$ requires that net transfers $\sum_{\omega \in \Omega_{f}} t_{\omega}$ be bounded below over all transfer vectors $t$ that are acceptable alongside any set $\Xi$ of trades. If a firm is willing to accept some set of trades alongside arbitrarily negative net transfers, then BCV fails. BCV is a weak assumption that is likely to be satisfied in any real-world economy. ${ }^{16}$ In particular, BCV is satisfied in Examples 1 and 2. Note that BCV allows for technological constraints, in that it permits sets of trades to be so undesirable to a firm that they remain less desirable than autarky regardless of how much the firm receives in net transfers.

Competitive equilibria may not exist under FS if BCV is not satisfied.

EXAMPLE 3-Competitive Equilibria Need Not Exist Under FS Alone: Consider two firms, $b$ and $s$, and one trade $\omega$ between them with $\mathrm{s}(\omega)=s$ and $\mathrm{b}(\omega)=b$. Suppose that $s$ is not willing to sell $\omega$ at any (finite) price, but $b$ would buy $\omega$ at any (finite) price. Note that the market does not clear at any price- $b$ always demands $\omega$ and $s$ never demands $\omega$. The issue is that the variation needed to compensate $b$ for going from autarky to trade is $-\infty$. If $b$ 's compensating variation were $-p$, then autarky could be sustained in equilibrium at any price above $p$.

On the other hand, FS and BCV together ensure that competitive equilibria exist.

\footnotetext{
${ }^{15}$ Our equivalence results are analogues of those in Hatfield et al. (2019) but apply even in environments with non-quasilinear utility functions. Just as several proofs in Hatfield et al. (2013) use the equivalence between the definitions of full substitutability proposed by Hatfield et al. (2019), several of our proofs use the equivalence between the definitions of full substitutability introduced in Appendix A.

${ }^{16}$ If transfers are denominated in different currencies, then BCV may only be satisfied after redenomination. For example, suppose that some trades are denominated in dollars and others in pounds, and that $£ 1=\$ 2$. If there are no transaction taxes, then firms will be willing to sustain a $-\$ 3 M$ transfer alongside a $£ 2 M$ transfer for all $M>0-$ a violation of $\mathrm{BCV}$. But $\mathrm{BCV}$ can be satisfied if the pound-denominated trades are redenominated in dollars.
} 


\section{THEOREM 1: Under FS and BCV, competitive equilibria exist.}

Theorem 1 generalizes the existence results of Kelso and Crawford (1982) and Hatfield et al. (2013). Unlike Kelso and Crawford (1982), we allow for a trading network structure. Unlike Hatfield et al. (2013), we allow utility to be imperfectly transferable between firms.

Our proof of Theorem 1 proceeds in three steps. First, we modify firms' preferences to bound their willingness to pay for trades. Second, we use BCV to show that every competitive equilibrium in the modified economy is in fact a competitive equilibrium in the original economy. Third, we use FS to construct a competitive equilibrium in the modified economy and complete the proof. Our overall strategy is similar to the strategy that Hatfield et al. (2013) employed to prove their existence result (Theorem 1 in Hatfield et al. (2013)). However, our arguments for the first and second steps are novel—we cannot apply the corresponding reasoning from Hatfield et al. (2013) because utility is imperfectly transferable in our model.

We now describe each of the steps in the proof of Theorem 1 in more detail.

Step 1: We construct a modified economy by giving all firms options to execute all trades at very undesirable prices. Specifically, we give every firm the option to execute any trade by paying a cost of $\Pi$. (We choose the value of $\Pi$ in Step 2.) Hence, firms have bounded willingness to pay for all trades in the modified economy (in a sense that we make precise in Section 5.4). We show that introducing these options preserves full substitutability.

Hatfield et al. (2013) applied a related-but not analogous-transformation in the proof of their existence result. Specifically, Hatfield et al. (2013) gave firms both the option to execute a trade by paying a cost of $\Pi$ and the option to dispose of an undesired trade for a cost of $\Pi$. However, the Hatfield et al. (2013) approach does not generally preserve full substitutability when forms of transfer are imperfectly substitutable.

Step 2: Using BCV, we choose $\Pi$ to ensure that all competitive equilibria in the modified economy are in fact competitive equilibria in the original economy. Specifically, we set

$$
\Pi=1-\sum_{f \in F} \inf _{(\Xi, t) \mid u^{f}(\Xi, t) \geq u^{f}(\emptyset, 0)} \sum_{\omega \in \Omega_{f}} t_{\omega},
$$

which is finite due to $\mathrm{BCV}$. With this choice of $\Pi$, firms do not use the options to execute trades in any competitive equilibrium in the modified economy. Indeed, $\Pi$ exceeds the total surplus in the economy, and hence if an option were used in a competitive equilibrium, then some firm would have to be worse off in the equilibrium than under autarky.

In contrast, Hatfield et al. (2013) chose $\Pi$ to be greater than the sum of the maximum absolute values of all firms' valuations. We cannot apply this approach directly because firms' preferences cannot generally be described by valuations of bundles of trades in our model.

Step 3: To complete the proof, we show that competitive equilibria exist in the modified economy. Our argument for this step follows familiar arguments from Crawford and Knoer (1981) and Kelso and Crawford (1982). Specifically, we discretize prices and use a generalized Deferred Acceptance algorithm (Ostrovsky (2008), Hatfield and Kominers (2012), Fleiner et al. (2018)) to show the existence of approximate equilibria in the modified economy, and then take limits to obtain a competitive equilibrium. This argument rests crucially on the fact (from Step 1) that in the modified economy, full substitutability is satisfied and firms have bounded willingness to pay for trades. 


\section{COMPETITIVE EQUILIBRIUM AND TRAIL STABILITY}

We now study the relationships between competitive equilibria and cooperative solution concepts from matching theory. Instead of assuming that firms are price-takers, we allow firms to recontract while keeping or dropping existing contracts.

A common restriction in cooperative solution concepts in the matching literature is individual rationality, which requires that no firm want to unilaterally drop any realized contract.

DEFINITION 2-Roth (1984), Hatfield et al. (2013): An outcome $A \subseteq X$ is individually rational if $A_{f} \in C^{f}\left(A_{f}\right)$ for all $f \in F$.

\subsection{Instability of Competitive Equilibrium}

One cooperative solution concept in the matching literature is stability (Roth (1984), Hatfield and Milgrom (2005), Hatfield and Kominers (2012)). At a stable outcome, there is no block - that is, no group of firms that can commit to recontracting among themselves while being free to drop any contracts. Hatfield et al. (2013) extended the definition of stability to settings with indifferences.

DEFinition 3-Hatfield et al. (2013): A non-empty set of contracts $Z \subseteq X \backslash A$ blocks an outcome $A$ if, for all $f \in F$ and $Y \in C^{f}\left(A_{f} \cup Z_{f}\right)$, we have that $Z_{f} \subseteq Y$. An outcome is stable if it is individually rational and unblocked.

Unfortunately, competitive equilibria may be unstable in the presence of frictions; moreover, stable outcomes need not exist even when competitive equilibria do.

EXAMPLE 4-Stable Outcomes Need Not Exist When Competitive Equilibria Exist: Consider the trading network from Example 2. We claim that there are no stable outcomes. Indeed, note that the autarky outcome-which is the unique competitive equilibrium outcome-is unstable because it is blocked by trade between $f_{1}$ and $f_{2}$. Note also that $f_{1}$ and $f_{3}$ cannot trade in any individually rational outcome due to the technological constraints faced by $f_{1}$ and $f_{2}$.

On the other hand, every individually rational outcome that involves trade between $f_{1}$ and $f_{2}$ is blocked by trade between $f_{1}$ and $f_{3}$. To see why, note that trade $\zeta$ cannot be realized at any price greater than $\$ 200$ in an individually rational outcome, as the surplus generated by trade between $f_{1}$ and $f_{2}$ is only $\$ 20$ and making a transfer of more than $\$ 200$ requires paying a transaction tax of more than $\$ 20$. But every outcome in which the trade $\zeta$ is realized at a price of at most $\$ 200$ is blocked by any contract $\left(\zeta^{\prime}, p_{\zeta^{\prime}}\right)$ with $200<p_{\zeta^{\prime}}<300 .^{17}$

Given the non-existence of stable outcomes and the instability of competitive equilibria, stability may be too stringent of a solution concept in general networks. We therefore turn to another cooperative solution concept.

\footnotetext{
${ }^{17}$ An alternative proof that no stable outcomes exist can be given using one of our results-Theorem E.1 in the Supplemental Material-which guarantees that all stable outcomes are competitive equilibrium outcomes under FS and BCV. Indeed, note that the autarky outcome is not stable. However, any stable outcome must be a competitive equilibrium outcome by Theorem E.1, and we showed in Example 2 that trade does not occur in any competitive equilibrium.
} 


\subsection{Trail Stability}

Trail stability is an extension of pairwise stability (in the sense of Gale and Shapley (1962)) to trading networks (Fleiner et al. (2018)). A trail is a sequence of contracts such that the buyer of each contract in the sequence (except for the last) is the seller of the next contract. A trail may involve a firm more than once and can begin and end with contracts that involve the same firm.

DEFINITION 4: A sequence of contracts $\left(x_{1}, \ldots, x_{n}\right)$ is a trail if $\mathrm{b}\left(x_{i}\right)=\mathrm{s}\left(x_{i+1}\right)$ for all $1 \leq i \leq n-1$.

Trail-stable outcomes are immune to sequential deviations called locally blocking trails. A locally blocking trail begins with a firm $f_{1}$ offering a sale contract $z_{1}$ that it wishes to sign given its existing contracts, possibly while dropping some existing contracts. The buyer $f_{2}$ may accept the offered contract $z_{1}$ while dropping some of its existing contracts, in which case a locally blocking trail is formed. The buyer may also hold the proposal $z_{1}$ and offer an additional sale contract $z_{2}$ to the original proposer or to another firm. This trail of linked offers $z_{1}, \ldots, z_{n}$ continues until a firm $f_{n+1}$ accepts an offered contract $z_{n}$ without offering another sale contract, in which case a locally blocking trail is formed. ${ }^{18}$

Our formal definition of trail stability extends the definition given by Fleiner et al. (2018) to settings with indifferences.

Definition 5: A trail $\left(z_{1}, \ldots, z_{n}\right) \in(X \backslash A)^{n}$ locally blocks an outcome $A$ if:

- $z_{1} \in Y \backslash A$ for all $Y \in C^{f_{1}}\left(A_{f_{1}} \cup\left\{z_{1}\right\}\right)$, where $f_{1}=\mathrm{s}\left(z_{1}\right)$;

- for $1 \leq i \leq n-1$, we have that $\left\{z_{i}, z_{i+1}\right\} \subseteq Y \backslash A$ for all $Y \in C^{f_{i+1}}\left(A_{f_{i+1}} \cup\left\{z_{i}, z_{i+1}\right\}\right)$, where $f_{i+1}=\mathrm{b}\left(z_{i}\right)=\mathrm{s}\left(z_{i+1}\right)$; and

- $z_{n} \in Y \backslash A$ for all $Y \in C^{f_{n+1}}\left(A_{f_{n+1}} \cup\left\{z_{n}\right\}\right)$, where $f_{n+1}=\mathrm{b}\left(z_{n}\right)$.

Such a trail is called a locally blocking trail. An outcome is trail-stable if it is individually rational and there is no locally blocking trail.

A trail locally blocks an individually rational outcome if, at each point at which a trail passes through a firm, the firm would like the one or two contracts that are available to it locally in the trail (when given access to the existing contracts). Intuitively, one should think of contracts in a locally blocking trail as being proposed via telephone by a manager at one firm to a manager at another (Fleiner et al. (2018)). If the sequence of phone conversations returns to a firm, a different manager (e.g., one from another division) answers the phone and considers the latest offer. Her decisions are independent of the offers received and made by other managers. Any manager's unilateral decision to accept an offered contract completes a locally blocking trail.

\subsection{A Cooperative Interpretation of Competitive Equilibria}

The main result of this section provides a cooperative interpretation of competitive equilibrium that holds even in the presence of frictions.

THEOREM 2: Every competitive equilibrium outcome is trail-stable.

\footnotetext{
${ }^{18}$ Note that locally blocking trails can also develop in the reverse direction, with firms making offers to buy instead of offers to sell.
} 
Theorem 2 implies that firms cannot improve upon competitive equilibrium outcomes by deviating along trails. In light of Theorem 2, every prediction of our model that holds in all trail-stable outcomes must also hold in all competitive equilibria.

To understand the intuition behind Theorem 2, consider any competitive equilibrium and any trail. In order for sellers to want to propose the contracts in the trail, the prices of all trades in the trail must be greater than their equilibrium prices. But the last buyer will only accept an offer if the price in the last contract is lower than the equilibrium price of the corresponding trade. Hence, there cannot be any locally blocking trails. The proof of Theorem 2 simply formalizes the preceding intuition.

As distortionary frictions can make competitive equilibria Pareto-inefficient, trailstable outcomes can also be Pareto-inefficient in light of Theorem 2-despite being defined cooperatively. ${ }^{19}$

EXAMPLE 5-A Pareto-Inefficient Trail-Stable Outcome: Consider the autarky outcome in the trading network from Example 1. As the autarky outcome is a competitive equilibrium outcome, it must be trail-stable by Theorem 2 .

More concretely, we show that there are no locally blocking trails for the autarky outcome. Consider an arbitrary trail $\left(z_{1}, \ldots, z_{n}\right)$. By construction, we must either have that $z_{1}=\left(\zeta, p_{\zeta}\right)$ or that $z_{1}=\left(\psi, p_{\psi}\right)$. In the former case, note that $f_{1}=\mathrm{s}\left(z_{1}\right)$ and that $\emptyset \in C^{f_{1}}\left(\left\{z_{1}\right\}\right)$ because $f_{1}$ is unwilling to trade $\zeta$ on its own-at any price. Hence, $\left(z_{1}, \ldots, z_{n}\right)$ cannot be a locally blocking trail. Analogous logic applies if $z_{1}=\left(\psi, p_{\psi}\right)$, because $f_{2}$ is unwilling to execute $\psi$ on its own. Alternatively, one could note that neither firm would be willing to accept a purchase (downstream) contract on its own, so $\emptyset \in C^{\mathrm{b}\left(z_{n}\right)}\left(\left\{z_{n}\right\}\right)$ holds for all contracts $z_{n}$.

But the autarky outcome is Pareto-inefficient because there are gains from trade between $f_{1}$ and $f_{2}$. This Pareto inefficiency arises in Example 2 as well.

Theorems 1 and 2 yield sufficient conditions for the existence of trail-stable outcomes: trail-stable outcomes exist under our conditions for the existence of competitive equilibria. ${ }^{20}$

\section{COROLLARY 1: Under FS and BCV, trail-stable outcomes exist.}

\subsection{A Competitive Interpretation of Trail Stability}

We now develop a competitive interpretation of trail stability. Formally, we say that an outcome $A$ lifts to a competitive equilibrium if $A$ is a competitive equilibrium outcomethat is, if $A$ can be supported by competitive equilibrium prices. As an outcome specifies prices only for the realized trades, the non-trivial part of lifting an outcome to a competitive equilibrium is constructing equilibrium prices for the unrealized trades.

Hatfield et al. (2013) showed that stable outcomes need not lift to competitive equilibria if FS is not satisfied. Using an example from Hatfield et al. (2013), we show that trail-stable outcomes do not generally lift to competitive equilibria either when FS is not satisfied-even when frictions and technological constraints are absent.

\footnotetext{
${ }^{19}$ As Blair (1988) showed, (pairwise) stable outcomes can be Pareto-inefficient even in two-sided many-tomany matching markets.

${ }^{20}$ Corollary 1 is a version of Theorem 1 in Fleiner et al. (2018)—which generalizes Theorem 1 in Ostrovsky (2008) from supply chains to general networks-for settings with prices that are continuous and potentially unbounded.
} 


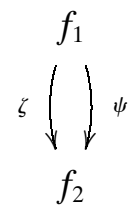

FIGURE 2.-Trading in Example 6. Arrows point from sellers to buyers.

EXAMPLE 6-Trail-Stable Outcomes Need Not Lift to Competitive Equilibria Without FS: Our example follows Example 1 in Hatfield et al. (2013). As depicted in Figure 2, there are two firms, $f_{1}$ and $f_{2}$, which interact via two trades. There are no taxes. The firms have quasilinear utility functions (see (1)) with valuation functions defined by

$$
\begin{array}{r}
v^{f_{1}}(\emptyset)=v^{f_{2}}(\emptyset)=0, \\
v^{f_{1}}(\{\zeta\})=v^{f_{1}}(\{\psi\})=v^{f_{1}}(\{\zeta, \psi\})=-4, \\
v^{f_{2}}(\{\zeta\})=v^{f_{2}}(\{\psi\})=v^{f_{2}}(\{\zeta, \psi\})=3 .
\end{array}
$$

Hatfield et al. (2013) showed that the autarky outcome is stable. We claim that the autarky outcome is also trail-stable. Indeed, as all trails consist of single contracts, we only need to show that there are no blocks that consist of one contract. Note that the seller, $f_{1}$, is only willing to offer a sale contract at a price above $\$ 4$, while the buyer, $f_{2}$, would not accept a single purchase contract at any price above $\$ 3$. Hence, there are no blocks that consist of single contracts.

However, the autarky outcome does not lift to a competitive equilibrium, as Hatfield et al. (2013) showed. To see why, consider any arrangement $[\emptyset ; p]$. If $\emptyset \in D^{f_{2}}(p)$, we must have that $p_{\zeta}, p_{\psi} \geq 3$. But it follows that $p_{\zeta}+p_{\psi}>4$, so $\emptyset \notin D^{f_{1}}(p)$.

Note that FS is not satisfied in this example because $f_{1}$ regards the two trades-which are both sales for $f_{1}$-as complements.

It turns out that FS is generally not sufficient for trail-stable outcomes to lift to competitive equilibria. Indeed, the following example shows that trail-stable outcomes may not lift to competitive equilibria even when FS and BCV are satisfied.

EXAMPLE 7-Trail-Stable Outcomes Need Not Lift to Competitive Equilibria Under FS and BCV: Consider the trading network from Example 1, but suppose that there are no transaction taxes $(\lambda=0 \%)$. The argument from Example 5 shows that no trail locally blocks the autarky outcome, and hence the autarky outcome is trail-stable. As there are gains from trade, the autarky outcome is Pareto-inefficient. However, as utility is perfectly transferable, all competitive equilibrium outcomes are Pareto-efficient (by the First Welfare Theorem). In particular, the autarky outcome cannot lift to a competitive equilibrium.

In Example 7, both firms face hard technological constraints: they are unwilling to execute any trade individually at any finite price, but would like to complete both trades together. The autarky outcome is trail-stable because neither the buyer nor the seller is willing to offer to buy or sell a single trade at any finite price.

To ensure that trail-stable outcomes lift to a competitive equilibrium, we impose a different regularity condition from $\mathrm{BCV}$. Intuitively, we require that firms have bounded willingness to pay for each trade. 
ASSUMPTION 3-Bounded Willingness to Pay-BWP: There exists $M$ such that for all firms $f \in F$ and all finite sets of contracts $Y, Z \subseteq X_{f}$ with $Z \in C^{f}(Y)$ :

- If $\left(\omega, p_{\omega}\right) \in Z_{\rightarrow f}$, then $p_{\omega}<M$.

- If $\left(\omega, p_{\omega}\right) \in Z_{f \rightarrow}$, then $p_{\omega}>-M$.

BWP requires that no firm be willing to pay $M$ or more for any trade-that is, that no firm be willing to buy any trade at a price $M$ or more or sell any trade at a price $-M$ or less. Note that BWP rules out many forms of technological constraints, including ones that are permitted under BCV and by Hatfield et al. (2013). In particular, BWP does not allow a firm to require a particular input in order to produce an output, as such constraints would make a firm willing to pay arbitrarily high prices for the input if the firm were able to procure arbitrarily high prices for the output. However, BWP allows for capacity constraints, as they never make trades desirable at extremely unfavorable prices.

BWP helps ensure that trail-stable outcomes lift to competitive equilibria. ${ }^{21}$

\section{THEOREM 3: Under FS and BWP, trail-stable outcomes lift to competitive equilibria.}

Theorem 3 provides a competitive interpretation of trail stability: every trail-stable outcome is consistent with price-taking equilibrium behavior by all firms (at least under FS and BWP). In light of Theorem 3, every prediction of our model that holds in all competitive equilibria must also hold in all trail-stable outcomes.

To prove Theorem 3, we adapt Kelso and Crawford's (1982) argument showing that stable outcomes are competitive equilibrium outcomes in two-sided many-to-one matching markets to our trading network setting. Kelso and Crawford (1982, p. 1487) set the prices of unrealized trades at the highest levels at which their sellers remain (weakly) unwilling to supply the trades given the prices of other trades. ${ }^{22}$ In two-sided many-to-one matching markets, the prices at which unit-demand sellers are willing to supply trades depend only on the prices of realized trades. The difficulty in extending Kelso and Crawford's argument is that, in trading networks, the prices at which sellers are willing to supply trades also depend on the prices of unrealized complementary input trades (which in turn need to be constructed in the course of the proof ).

We therefore determine whether a $\operatorname{seller} s(z)$ desires a contract $z$ jointly with a trail of proposals that terminates in an input contract for $\mathrm{s}(z)$ that is complementary to $z$. More precisely, we consider trails in which sellers are willing to propose each contract (when given access to the preceding contract in the trail) but where the last contract $z$ in the trail may not be acceptable to its buyer $\mathrm{b}(z)$. We call such a trail locally semi-blocking, as it would be locally blocking if $z$ were desirable to $\mathrm{b}(z) \cdot{ }^{23}$ Note that in two-sided markets, the locally semi-blocking trails are simply the trails that consist of a single contract that is desirable to its seller. Therefore, analogously to Kelso and Crawford (1982), we set the price of an unrealized trade to be the highest price at which it does not appear in any locally semi-blocking trail. We then replace infinite prices by large, finite values

\footnotetext{
${ }^{21}$ Despite the fact that BWP is not satisfied in Examples 1 and 2, trail-stable outcomes lift to competitive equilibria in both examples. Thus, BWP is sufficient but not necessary for trail-stable outcomes to lift to competitive equilibria.

${ }^{22}$ Stability ensures that buyers are (weakly) unwilling to demand the unrealized trades at the constructed candidate equilibrium prices, as Kelso and Crawford (1982) showed.

${ }^{23}$ Fleiner et al. (2018) used the concept of locally semi-blocking trails to provide a correspondence between trail-stable outcomes and fixed points of a generalized Deferred Acceptance algorithm in trading networks with discrete and bounded prices.
} 


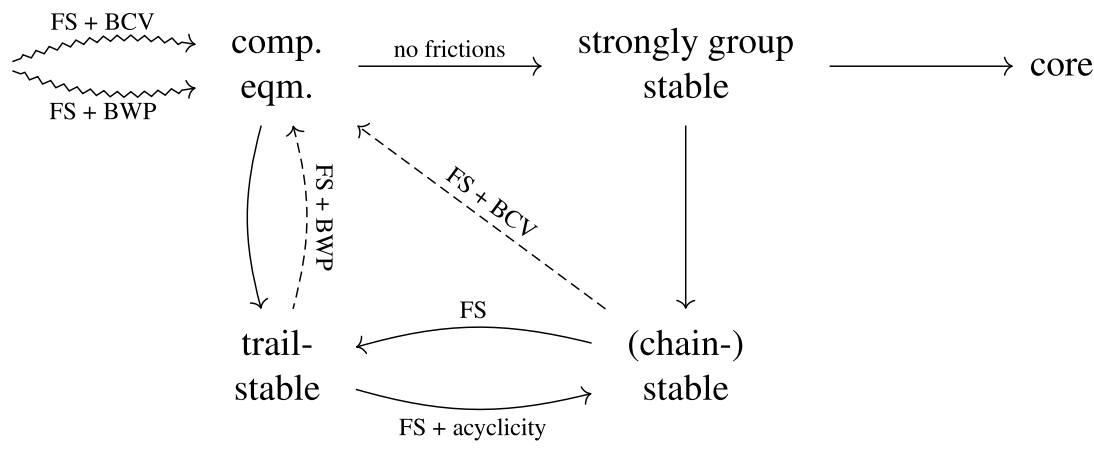

FIGURE 3.-Summary of our results. The squiggly arrows represent existence results, the ordinary arrows represent relationships between solution concepts, and the dashed arrows represent lifting results. We label arrows by the hypotheses of the corresponding results. The "no frictions" condition is defined in Appendix E in the Supplemental Material.

(using BWP) and use FS and trail stability to prove that the constructed prices are in fact competitive equilibrium prices. ${ }^{24}$

Theorems 2 and 3 imply that competitive equilibria are essentially equivalent to trailstable outcomes in our model under FS and BWP..$^{25}$

COROLLARY 2: Under FS and BWP, competitive equilibrium outcomes and trail-stable outcomes exist and coincide.

Corollary 2 provides competitive foundations for trail stability and cooperative foundations for competitive equilibrium: the assumption that firms coordinate on a trail-stable outcome produces the same predictions as the assumption that firms act as price-takers in equilibrium.

\section{OTHER COOPERATIVE SOLUTION CONCEPTS}

We have thus far focused on competitive equilibria and trail stability in trading networks with frictions. In addition to stability and trail stability, other cooperative solution concepts for trading networks that have been proposed in the matching literature include chain stability (Ostrovsky (2008), Hatfield et al. (2018)) and strong group stability (Hatfield et al. (2013)). Chain stability weakens stability by only allowing firms to deviate along blocking chains of contracts, while strong group stability strengthens stability by allowing firms to commit to deviations that are not individually rational. In this section, we describe the relationships between trail stability, chain stability, stability, and strong group stability. We present the definitions and results formally in Appendix E in the Supplemental Material. Figure 3 summarizes our results.

First, under FS, every stable or chain-stable outcome is trail-stable (see also Fleiner et al. (2018)). Therefore, stability and chain stability are strictly stronger cooperative solution concepts than trail stability under our sufficient conditions for the existence of

\footnotetext{
${ }^{24}$ Hatfield et al. (2013) took a different approach to showing that stable outcomes lift to competitive equilibria in their transferable utility framework (Theorem 6 in Hatfield et al. (2013)). Specifically, they exploited the existence and efficiency of competitive equilibria in an auxiliary economy. We cannot use their approach because frictions make competitive equilibria inefficient in general in our model.

${ }^{25}$ To derive Corollary 2 formally, we need to establish that competitive equilibria exist under FS and BWP, as Theorem B.1 in the Appendix shows.
} 
competitive equilibria and trail-stable outcomes. ${ }^{26}$ Furthermore, stable and chain-stable outcomes lift to competitive equilibria under FS and BCV. Hence, the lifting results of Hatfield et al. $(2013,2018)$ continue to hold even in the presence of distortionary frictions and income effects-unlike the existence results for stability and chain stability, which do not generally hold in our model. ${ }^{27}$

Second, stable and chain-stable outcomes exist and coincide with trail-stable outcomes and competitive equilibrium outcomes under FS and BCV in vertical supply chains. Hence, the results of Ostrovsky (2008) and Hatfield and Kominers (2012) on the existence of chain-stable and stable outcomes in supply chains with discrete and bounded prices extend to our setting - which features continuous and unbounded prices.

Finally, in trading networks without distortionary frictions, stable, chain-stable, and strongly group stable outcomes exist and coincide with competitive equilibrium outcomes under FS and BCV. Hence, the results of Hatfield et al. $(2013,2018)$ on the equivalence between stability, chain stability, strong group stability, and competitive equilibrium persist in the presence of income effects. Under FS and BWP, all of these solution concepts are also equivalent to trail stability.

\section{CONCLUSION}

This paper develops a model of differentiated markets with frictions based on matching in trading networks. Competitive equilibria exist in our model when trades are fully substitutable (and mild regularity conditions are satisfied) but may be inefficient. ${ }^{28}$ In the presence of frictions, competitive equilibria may be unstable but still essentially coincide with trail-stable outcomes.

Taken as a whole, our results provide a relationship between competitive and cooperative solution concepts in differentiated markets that applies even in the presence of frictions. Our competitive interpretation of trail stability guarantees that, as long as firms coordinate on a trail-stable outcome, they act as if they take prices as given. Hence, even if price-taking is not a reasonable assumption per se (e.g., in thin markets), it is actually a consequence of a form of cooperative behavior. On the other hand, our cooperative interpretation of competitive equilibrium guarantees that firms cannot improve upon equilibrium outcomes by deviations along trails. Therefore, even if it is difficult for firms to coordinate with each other (e.g., in thick markets), any equilibrium outcome will be trailstable as long as firms take prices as given. In light of our equivalence result, equilibrium analysis can be performed using scale-independent solution concepts, even in markets with frictions.

We conclude by leaving two open questions. First, to what extent can the condition that firms have bounded willingness to pay for trades be relaxed while still ensuring that trail-stable outcomes lift to competitive equilibria? Second, can externalities (as analyzed by Pycia and Yenmez (2017) and Rostek and Yoder (2018)) be incorporated into our analysis?

\footnotetext{
${ }^{26}$ Note, however, that stable and chain-stable outcomes are generally not trail-stable without FS.

${ }^{27}$ In Example 4, we showed that there are no stable outcomes in Example 2. Similar logic shows that there are no chain-stable outcomes in that example either.

${ }^{28}$ Subsequent to our work, Schlegel (2019) established results on the lattice structure of the set of competitive equilibria.
} 


\section{APPENDIX A: AN EQUIVALENT DEFINITION OF Full SubSTITUTABILITY}

In this appendix, we show that full substitutability is equivalent to full substitutability including indifferences - a condition that deals with indifferences more explicitly-as well as to the weak quasisubmodularity of the indirect utility function. We use the equivalence between full substitutability and full substitutability including indifferences in the proof of Theorem 3, and the equivalence between full substitutability and the weak quasisubmodularity of the indirect utility function in the proof of Theorem 1. Our equivalence result is a version of Theorems 2 and A.1 in Hatfield et al. (2019) that does not assume that preferences are quasilinear.

Full substitutability including indifferences combines four conditions, which are each similar to conditions defined in Appendix A in Hatfield et al. (2019). The first condition, increasing price full substitutability for sales, requires that sales be substitutable to each other and complementary to purchases as prices rise (i.e., as the set of available purchases shrinks and the set of available sales expands). The analogous condition for purchases is decreasing price full substitutability for purchases. We also consider two other similar conditions, decreasing price full substitutability for sales and increasing price full substitutability for purchases, which are not exactly analogous to the first two conditions due to the possibility of income effects in our model.

ASSUMPTION A.1-Full Substitutability Including Indifferences (FSII): Let $f \in F$ be a firm and let $Y, Y^{\prime} \subseteq X_{f}$ be finite sets of contracts. For all $Z \in C^{f}(Y)$ :

- Increasing price full substitutability for sales (IFSS): If we have that $Y_{\rightarrow f} \supseteq Y_{\rightarrow f}^{\prime}$ and that $Y_{f \rightarrow \subseteq} Y_{f \rightarrow}^{\prime}$, then there exists $Z^{\prime} \in C^{f}\left(Y^{\prime}\right)$ with $Z^{\prime} \cap Y_{f \rightarrow} \subseteq Z$.

- Decreasing price full substitutability for purchases (DFSP): If we have that $Y_{f \rightarrow \supseteq Y_{f \rightarrow}^{\prime}}$ and that $Y_{\rightarrow f} \subseteq Y_{\rightarrow f}^{\prime}$, then there exists $Z^{\prime} \in C^{f}\left(Y^{\prime}\right)$ with $Z^{\prime} \cap Y_{\rightarrow f} \subseteq Z$.

For all $y \in Y$ such that there exists $Z \in C^{f}(Y)$ with $y \in Z$ :

- Decreasing price full substitutability for sales (DFSS): If we have that $Y_{\rightarrow f} \subseteq Y_{\rightarrow f}^{\prime}$ and

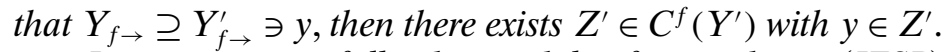

- Increasing price full substitutability for purchases (IFSP): If we have that $Y_{f \rightarrow} \subseteq Y_{f \rightarrow}^{\prime}$ and that $Y_{\rightarrow f} \supseteq Y_{\rightarrow f}^{\prime} \ni y$, then there exists $Z^{\prime} \in C^{f}\left(Y^{\prime}\right)$ with $y \in Z^{\prime}$.

To define our weak quasisubmodularity condition, we use infinite prices to denote unavailable trades. Formally, we define a set of prices by

$$
\mathbf{P}^{f}=(\mathbb{R} \cup\{-\infty\})^{\Omega_{f \rightarrow}} \times(\mathbb{R} \cup\{\infty\})^{\Omega_{\rightarrow f}},
$$

Firm $f$ 's indirect utility function $V^{f}: \mathrm{P}^{f} \rightarrow \mathbb{R}$ is defined by

$$
V^{f}(p)=\max _{\Xi \subseteq \Omega_{f}} u^{f}\left(\Xi,\left(p_{\Xi_{f \rightarrow}},(-p)_{\Xi_{\rightarrow f}}, 0_{\Omega_{f} \backslash \Xi}\right)\right) .
$$

Recall that the indirect utility function $V^{f}$ is submodular if the inequality

$$
V^{f}(p)+V^{f}(q) \geq V^{f}(p \vee q)+V^{f}(p \wedge q)
$$

holds for all $p, q \in \mathrm{P}^{f}$. When utility functions are quasilinear, Hatfield et al. (2019) showed that full substitutability is equivalent to the submodularity of the indirect utility function. Without quasilinearity, full substitutability does not generally entail the submodularity of the indirect utility function, as Hatfield and Kominers (2012) showed (see also 
Hatfield, Jagadeesan, and Kominers (2019)). We therefore adapt the weak quasisubmodularity condition of Hatfield, Jagadeesan, and Kominers (2019) to obtain a characterization of full substitutability in terms of the indirect utility function. ${ }^{29}$

AssumPTION A.2-Weak Quasisubmodularity (WQ): For all $f \in F$ and $p, q \in \mathrm{P}^{f}$, if $p_{\Omega_{f \rightarrow}} \leq q_{\Omega_{f \rightarrow}}$ or $p_{\Omega_{\rightarrow f}} \leq q_{\Omega_{\rightarrow f}}$, then

$$
\begin{array}{lll}
V^{f}(p)<V^{f}(p \wedge q) & \Longrightarrow & V^{f}(p \vee q)<V^{f}(q), \\
V^{f}(p \vee q)>V^{f}(q) & \Longrightarrow & V^{f}(p)>V^{f}(p \wedge q) .
\end{array}
$$

To understand the relationship between weak quasisubmodularity and quasisubmodularity, recall that quasisubmodularity (in the sense of Milgrom and Shannon (1994)) requires that (A.1) hold for all $p, q \in \mathrm{P}^{f}$. Weak quasisubmodularity weakens quasisubmodularity by only requiring that (A.1) hold for price vectors $p, q$ with $p_{\Omega_{f \rightarrow}} \leq q_{\Omega_{f \rightarrow}}$ or $p_{\Omega_{\rightarrow f}} \leq q_{\Omega_{\rightarrow f}}$.

The main result of this appendix asserts that full substitutability, full substitutability including indifferences, and weak quasisubmodularity are all equivalent.

THEOREM A.1: The conditions FS, FSII, WQ are all equivalent.

We prove Theorem A.1 in Appendix D in the Supplemental Material.

Although Hatfield et al. (2019) ruled out income effects, Theorem A.1 is logically independent of Hatfield et al.'s analogous results (Theorems 2 and A.1 in Hatfield et al. (2019)) as we derive weaker conclusions.

\section{APPENDIX B: PROOF OF THEOREM 1}

In the proof of Theorem 1, we need a result on the existence of competitive equilibria under FS and BWP.

THEOREM B.1: Under FS and BWP, competitive equilibria exist.

To prove Theorem 1, we first modify utility functions so BWP is satisfied (Lemma B.1), ensuring that our modification preserves FS (Lemma B.2). We next show that under BCV, every competitive equilibrium in the modified economy yields a competitive equilibrium in the original economy (Lemma B.4). We then prove Theorem B.1 and complete the proof of Theorem 1 by combining Theorem B.1 with Lemmata B.1, B.2, and B.4.

\section{B.1. The Modified Economy}

For $f \in F$, we define a quantity

$$
\mathcal{K}^{f}=-\inf _{(\Xi, t) \mid u^{f}(\Xi, t) \geq u^{f}(\emptyset, 0)} \sum_{\omega \in \Omega_{f}} t_{\omega},
$$

which is finite by BCV. Let $\Pi \geq 1+\sum_{f \in F} \mathcal{K}^{f}$ be an arbitrary real number.

\footnotetext{
${ }^{29}$ Hatfield, Jagadeesan, and Kominers (2019) corrected a result of Hatfield and Kominers (2012) that characterizes full substitutability in terms of the indirect utility function in trading networks with discrete and bounded prices.
} 
We modify the economy by giving firms the option to execute any trade for a cost of $\Pi .^{30}$ Formally, for $f \in F$, we define a utility function $\widehat{u}^{f}: \mathcal{P}\left(\Omega_{f}\right) \times \mathbb{R}^{\Omega_{f}} \rightarrow \mathbb{R} \cup\{-\infty\}$ by

$$
\widehat{u}^{f}(\Xi, t)=\max _{\Xi \subseteq \Psi \subseteq \Omega_{f}} u^{f}\left(\Psi,\left(t_{\Omega_{f} \backslash \Psi \cup \Xi},(t-\Pi)_{\Psi \backslash \Xi)}\right) .\right.
$$

The function $\widehat{u}^{f}(\Xi, t)$ is continuous in $t$ and satisfies the requisite monotonicity condition from Section 2.2.

Consider a modified economy in which utility functions are given by $\widehat{u}^{f}$ for $f \in F$; we show that BWP and FS hold in the modified economy. ${ }^{31}$ We first prove that BWP holds.

\section{LEMMA B.1: The modified economy satisfies BWP.}

Proof: We claim that BWP holds with $M=\Pi+1$. Let $f \in F$, let $\omega \in \Omega_{f} \backslash \Xi$, let $\Xi \subseteq \Omega_{f}$, and let $t \in \mathbb{R}^{\Omega_{f}}$ be such that $t_{\omega}=0$. Note that, for all $\Psi \supseteq \Xi$ with $\omega \in \Psi$, because $M>\Pi=\Pi-t_{\omega}$, we have that

$$
u^{f}\left(\Psi,\left(t_{\Omega_{f} \backslash \Psi \cup \Xi},(t-\Pi)_{\Psi \backslash \Xi \backslash\{\omega\}},(-M)_{\omega}\right)\right)<u^{f}\left(\Psi,\left(t_{\Omega_{f} \backslash \Psi \cup \Xi},(t-\Pi)_{\Psi \backslash \Xi}\right)\right)
$$

whenever $u^{f}\left(\Psi,\left(t_{\Omega_{f} \backslash \Psi \cup \Xi},(t-\Pi)_{\Psi \backslash \Xi \backslash\{\omega\}},(-M)_{\omega}\right)\right) \in \mathbb{R}$. Hence, we have that

$$
\begin{aligned}
\widehat{u}^{f}\left(\Xi \cup\{\omega\},\left(t_{\left.\Omega_{f} \backslash \omega \omega\right\}},(-M)_{\omega}\right)\right) & =\max _{\Xi \cup\{\omega\} \subseteq \Psi \subseteq \Omega_{f}} u^{f}\left(\Psi,\left(t_{\Omega_{f} \backslash \Psi \cup \Xi},(t-\Pi)_{\Psi \backslash \Xi \backslash \backslash \omega\}},(-M)_{\omega}\right)\right) \\
& <\max _{\Xi \cup\{\omega\rfloor \Psi \subseteq \Omega_{f}} u^{f}\left(\Psi,\left(t_{\Omega_{f} \backslash \Psi \cup \Xi},(t-\Pi)_{\Psi \backslash \Xi}\right)\right) \\
& \leq \max _{\Xi \subseteq \Psi \subseteq \Omega_{f}} u^{f}\left(\Psi,\left(t_{\Omega_{f} \backslash \Psi \cup \Xi},(t-\Pi)_{\Psi \backslash \Xi)}\right)\right) \\
& =\widehat{u}^{f}(\Xi, t)
\end{aligned}
$$

whenever $\widehat{u}^{f}\left(\Xi \cup\{\omega\},\left(t_{\Omega_{f} \backslash\{\omega\}},(-M)_{\omega}\right)\right) \in \mathbb{R}$. Therefore, firm $f$ will never choose a contract $\left(\omega, p_{\omega}\right)$ with $p_{\omega} \geq M$ (resp. $\left.p_{\omega} \leq-M\right)$ if $\omega \in \Omega_{\rightarrow f}$ (resp. $\omega \in \Omega_{f \rightarrow}$ ). Since $f, \omega, \Xi$, and $t$ were arbitrary, the claim follows.

We next prove that the modified economy satisfies FS. For this part of the argument, we use the equivalence between FS and WQ from Theorem A.1.

\section{LEMMA B.2: Under FS, the modified economy satisfies FS.}

PROOF: The proof of this claim is similar to the proof of Theorem 2 in Hatfield et al. (2019). Let $V^{f}, \widehat{V}^{f}: \mathrm{P}^{f} \rightarrow \mathbb{R} \cup\{-\infty\}$ denote the indirect utility functions for the utility

\footnotetext{
${ }^{30}$ Hatfield et al. (2019) showed that such trade endowments preserve full substitutability when preferences are quasilinear (see Theorem 2 in Hatfield et al. (2019)).

${ }^{31}$ The arguments that FS and BWP hold in the modified economy apply not only for $\Pi \geq 1+\sum_{f \in F} \mathcal{K}^{f}$, but rather for all values of $\Pi$. The lower bound on $\Pi$ is used in Lemma B.4 in Appendix B.2 to ensure that competitive equilibria in the modified economy are in fact competitive equilibria in the original economy. Therefore, BCV is not important for constructing a modified economy in which FS and BWP hold. Rather, $\mathrm{BCV}$ is crucial for constructing such a modified economy whose competitive equilibria are all competitive equilibria in the original economy.
} 
functions $u^{f}$ and $\widehat{u}^{f}$, respectively. Note that, for all $p \in \mathrm{P}^{f}$, we have that

$$
\begin{aligned}
\widehat{V}^{f}(p) & =\max _{\Xi \subseteq \Omega_{f}} \widehat{u}^{f}\left(\Xi,\left(p_{\Xi_{f \rightarrow}},(-p)_{\Xi_{\rightarrow f}}, 0_{\Omega_{f} \backslash \Xi}\right)\right) \\
& =\max _{\Xi \subseteq \Omega_{f}} \max _{\Xi \subseteq \Psi \subseteq \Omega_{f}} u^{f}\left(\Psi,\left(p_{\Xi_{f \rightarrow}},(-p)_{\Xi_{\rightarrow f}},(-\Pi)_{\Psi \backslash \Xi}, 0_{\Omega_{f} \backslash \Psi}\right)\right) .
\end{aligned}
$$

Letting $\Gamma=\Psi \backslash \Xi$, we have that

$$
\begin{aligned}
\widehat{V}^{f}(p) & =\max _{\Gamma \subseteq \Psi \subseteq \Omega_{f}} u^{f}\left(\Psi,\left(p_{\Psi_{f \rightarrow \mid}},(-p)_{\Psi_{\rightarrow f} \backslash \Gamma},(-\Pi)_{\Psi \cap \Gamma}, 0_{\Omega_{f} \backslash \Psi}\right)\right) \\
& =\max _{\Psi, \Gamma \subseteq \Omega_{f}} u^{f}\left(\Psi,\left(p_{\Psi_{f \rightarrow} \backslash \Gamma},(-p)_{\Psi_{\rightarrow f} \backslash \Gamma},(-\Pi)_{\Psi \cap \Gamma}, 0_{\Omega_{f} \backslash \Psi}\right)\right) \\
& =\max _{\Gamma \subseteq \Omega_{f}} V^{f}\left(p_{\Omega_{f} \backslash \Gamma}, \Pi_{\Gamma_{f \rightarrow}},(-\Pi)_{\Gamma_{\rightarrow f}}\right) .
\end{aligned}
$$

It follows that

$$
\widehat{V}^{f}(p)=V^{f}\left(\max \{p,-\Pi\}_{\Omega_{f \rightarrow}}, \min \{p, \Pi\}_{\Omega_{\rightarrow f}}\right) .
$$

We claim that the indirect utility function $\widehat{V}^{f}$ must be weakly quasisubmodular. Let $p, q \in \mathrm{P}^{f}$ and suppose that $p_{\Omega_{f \rightarrow}} \leq q_{\Omega_{f \rightarrow}}$ or that $p_{\Omega_{\rightarrow f}} \leq q_{\Omega_{\rightarrow f}}$. We define $p^{\prime}, q^{\prime} \in \mathrm{P}^{f}$ by

$$
p^{\prime}=\left(\max \{p,-\Pi\}_{\Omega_{f \rightarrow}}, \min \{p, \Pi\}_{\Omega_{\rightarrow f}}\right) \quad \text { and } \quad q^{\prime}=\left(\max \{q,-\Pi\}_{\Omega_{f \rightarrow}}, \min \{q, \Pi\}_{\Omega_{\rightarrow f}}\right) .
$$

By construction, we have $p_{\omega}^{\prime} \leq q_{\omega}^{\prime}$ whenever $p_{\omega} \leq q_{\omega}$. Hence, we have that $p_{\Omega_{f \rightarrow}}^{\prime} \leq q_{\Omega_{f \rightarrow}}^{\prime}$ or that $p_{\Omega_{\rightarrow f}}^{\prime} \leq q_{\Omega_{\rightarrow f}}^{\prime}$.

If $\widehat{V}^{f}(p)<\widehat{V}^{f}(p \wedge q)$, then (B.1) implies that

$$
V^{f}\left(p^{\prime}\right)=\widehat{V}^{f}(p)<\widehat{V}^{f}(p \wedge q)=V^{f}\left(p^{\prime} \wedge q^{\prime}\right) .
$$

Theorem A.1 guarantees that WQ holds, and it follows that $V^{f}\left(p^{\prime} \vee q^{\prime}\right)<V^{f}\left(q^{\prime}\right)$. Equation (B.1) hence implies that

$$
\widehat{V}^{f}(p \vee q)=V^{f}\left(p^{\prime} \vee q^{\prime}\right)<V^{f}\left(q^{\prime}\right)=\widehat{V}^{f}(q) .
$$

Hence, we have shown that $\widehat{V}^{f}(p)<\widehat{V}^{f}(p \wedge q) \Longrightarrow \widehat{V}^{f}(p \vee q)<\widehat{V}^{f}(q)$. Similar logic shows that $\widehat{V}^{f}(p \vee q)>\widehat{V}^{f}(q) \Longrightarrow \widehat{V}^{f}(p)>\widehat{V}^{f}(p \wedge q)$. Therefore, the modified economy satisfies WQ. By Theorem A.1, the modified economy must also satisfy FS. Q.E.D.

\section{B.2. Outcomes in the Modified Economy}

This subsection shows that competitive equilibria in the modified economy give rise to competitive equilibria in the original economy (Lemma B.4). The following lemma shows that firm $f$ can only produce $\mathcal{K}^{f}$ units of surplus in the modified economy and that the options to execute trades can only be used at a social cost of $\Pi$. As will be seen in the proof of Lemma B.4, it follows that the options to execute trades at a cost of $\Pi$ cannot be used in any competitive equilibria.

Lemma B.3: Let $\Xi \subseteq \Omega_{f}$ and $t \in \mathbb{R}^{\Omega_{f}}$. Suppose that $\widehat{u}^{f}(\Xi, t) \geq \widehat{u}^{f}(\emptyset, 0)$. Under BCV: 
(a) We have that $\sum_{\omega \in \Omega_{f}} t_{\omega} \geq-\mathcal{K}^{f}$.

(b) If $u^{f}(\Xi, t)<\widehat{u}^{f}(\Xi, t)$, then we have that $\sum_{\omega \in \Omega_{f}} t_{\omega} \geq \Pi-\mathcal{K}^{f}$.

ProOF: Note that $\widehat{u}^{f}(\emptyset, 0) \geq u^{f}(\emptyset, 0)$, and hence we have that $\widehat{u}^{f}(\Xi, t) \geq u^{f}(\emptyset, 0)$. Let $\Xi \subseteq \Psi \subseteq \Omega_{f}$ be such that

$$
\widehat{u}^{f}(\Xi, t)=u^{f}\left(\Psi,\left(t_{\Omega_{f} \backslash \Psi \cup \Xi},(t-\Pi)_{\Psi \backslash \Xi}\right)\right) .
$$

The definition of $\mathcal{K}^{f}$ implies that

$$
-\mathcal{K}^{f} \leq \sum_{\omega \in \Omega_{f} \backslash \Psi \cup \Xi} t_{\omega}+\sum_{\omega \in \Psi \backslash \Xi}\left(t_{\omega}-\Pi\right)=-\Pi \cdot|\Psi \backslash \Xi|+\sum_{\omega \in \Omega_{f}} t_{\omega},
$$

and hence we have that

$$
\Pi \cdot|\Psi \backslash \Xi|-\mathcal{K}^{f} \leq \sum_{\omega \in \Omega_{f}} t_{\omega} .
$$

As $|\Psi \backslash \Xi| \geq 0$, Part (a) follows from (B.2). If $u^{f}(\Xi, t)<\widehat{u}^{f}(\Xi, t)$, then we must have that $\Psi \neq \Xi$. As $|\Psi \backslash \Xi| \geq 1$ must hold in this case, Part (b) follows from (B.2) as well.

Q.E.D.

We now show that competitive equilibria in the modified economy give rise to competitive equilibria in the original economy.

LEMMA B.4: Under BCV, every competitive equilibrium in the modified economy is a competitive equilibrium in the original economy.

Proof: Let $[\Xi ; p]$ be a competitive equilibrium in the modified economy. For $f \in F$, let $t^{f}=\left(p_{\Xi_{f \rightarrow}},(-p)_{\Xi_{\rightarrow f}}, 0_{\Omega_{f} \backslash \Xi}\right)$. Since $[\Xi ; p]$ is a competitive equilibrium in the modified economy, we have that $\widehat{u}^{f}\left(\Xi_{f}, t^{f}\right) \geq \widehat{u}^{f}(\emptyset, 0)$ for all $f \in F$. Note that

$$
\sum_{f \in F} \sum_{\omega \in \Omega_{f}} t_{\omega}^{f}=\sum_{f \in F} \sum_{\omega \in \Xi_{f}} t_{\omega}^{f}=\sum_{\omega \in \Xi}\left(t_{\omega}^{s(\omega)}+t_{\omega}^{\mathrm{b}(\omega)}\right)=\sum_{\omega \in \Xi}\left(p_{\omega}-p_{\omega}\right)=0 .
$$

Hence, for all $f \in F$, we have that

$$
\sum_{\omega \in \Omega_{f}} t_{\omega}^{f}=-\sum_{f^{\prime} \in F \backslash\{f\}} \sum_{\omega \in \Omega_{f^{\prime}}} t_{\omega}^{f^{\prime}} \leq-\sum_{f^{\prime} \in F \backslash\{f\}} \mathcal{K}^{f^{\prime}} \leq \Pi-\mathcal{K}^{f}-1<\Pi-\mathcal{K}^{f},
$$

where the first inequality follows from Lemma B.3(a) and the second inequality is due to the definition of $\Pi$. By the contrapositive of Lemma B.3(b), it follows that $u^{f}\left(\Xi_{f}, t^{f}\right) \geq \widehat{u}^{f}\left(\Xi_{f}, t^{f}\right)$ for all $f \in F$. As $u^{f}\left(\Xi_{f}, t^{f}\right) \leq \widehat{u}^{f}\left(\Xi_{f}, t^{f}\right)$, we must have that $u^{f}\left(\Xi_{f}, t^{f}\right)=\widehat{u}^{f}\left(\Xi_{f}, t^{f}\right)$ for all $f \in F$.

Let $f \in F$ be arbitrary. For all $\Psi \subseteq \Omega_{f}$, we have that

$$
\begin{aligned}
u^{f}\left(\Xi_{f}, t^{f}\right)=\widehat{u}^{f}\left(\Xi_{f}, t^{f}\right) & \geq \widehat{u}^{f}\left(\Psi,\left(p_{\Psi_{f \rightarrow}},(-p)_{\Psi_{\rightarrow f}}, 0_{\Omega_{f} \backslash \Psi}\right)\right) \\
& \geq u^{f}\left(\Psi,\left(p_{\Psi_{f \rightarrow}},(-p)_{\Psi_{\rightarrow f}}, 0_{\Omega_{f} \backslash \Psi}\right)\right),
\end{aligned}
$$


where the first inequality holds because $[\Xi ; p]$ is a competitive equilibrium in the modified economy and the second inequality holds due to the definition of $\widehat{u}^{f}$. It follows that $\Xi_{f} \in D^{f}(p)$. As $f$ was arbitrary, $[\Xi ; p]$ must be a competitive equilibrium in the original economy.

Q.E.D.

\section{B.3. Proof of Theorem B.1}

Let $M$ be as in BWP. Intuitively, we consider a grid of size $\epsilon$ in $[-2 M, 2 M]^{\Omega}$, chosen so there are no indifferences. We then use the Gale-Shapley operator of Hatfield and Kominers (2012) and Fleiner et al. (2018) to produce an $\epsilon$-equilibrium. Taking limits as $\epsilon \rightarrow 0$, we obtain a competitive equilibrium.

Formally, we say that a vector $\delta \in(-\epsilon, \epsilon)^{\Omega}$ is $\epsilon$-regular if $D^{f}$ is single-valued on $[-2 M, 2 M]^{\Omega_{f}} \cap\left(\epsilon \mathbb{Z}^{\Omega_{f}}+\delta_{\Omega_{f}}\right)$ for all $f \in F$. The following claim asserts that the set of regular vectors is dense.

Claim B.1: For all $\epsilon>0$, the set of $\epsilon$-regular vectors is dense in $(-\epsilon, \epsilon)^{\Omega}$.

PROOF: For a firm $f \in F$, let

$$
\mathfrak{S}_{f}=\left\{p \in \mathbb{R}^{\Omega}|| D^{f}\left(p_{\Omega_{f}}\right) \mid=1\right\} .
$$

We claim that $\mathfrak{S}_{f}$ is open and dense in $\mathbb{R}^{\Omega_{f}}$. The set $\mathfrak{S}_{f}$ is open because $D^{f}$ is upper hemicontinuous (by Berge's Maximum Theorem) and non-empty-valued and $\mathcal{P}\left(\Omega_{f}\right)$ is discrete. To show that $\mathfrak{S}_{f}$ is dense, note that for all $\Xi \neq \Xi^{\prime} \subseteq \Omega_{f}$, the set

$$
\mathfrak{T}_{\Xi, \Xi^{\prime}}=\left\{\begin{array}{l|l}
p \in \mathbb{R}^{\Omega_{f}} & \begin{array}{c}
u^{f}\left(\Xi,\left(p_{\Xi_{f \rightarrow}},(-p)_{\Xi_{\rightarrow f}}, 0_{\Omega_{f} \backslash \Xi}\right)\right) \\
=u^{f}\left(\Xi^{\prime},\left(p_{\Xi_{f \rightarrow}^{\prime}},(-p)_{\Xi_{\rightarrow f}^{\prime}}, 0_{\Omega_{f} \backslash \Xi^{\prime}}\right)\right)
\end{array} \neq-\infty
\end{array}\right\}
$$

is nowhere dense. Indeed, if $p \in \mathfrak{T}_{\Xi, \Xi^{\prime}}$, then we have that

$$
u^{f}\left(\Xi,\left(p_{\Xi_{f \rightarrow}}^{\prime},\left(-p^{\prime}\right)_{\Xi_{\rightarrow f}}, 0_{\Omega_{f} \backslash \Xi}\right)\right) \neq u^{f}\left(\Xi^{\prime},\left(p_{\Xi_{f \rightarrow}^{\prime}}^{\prime},\left(-p^{\prime}\right)_{\Xi_{\rightarrow f}^{\prime}}, 0_{\Omega_{f} \backslash \Xi^{\prime}}\right)\right)
$$

for all price vectors $p^{\prime}=\left(p_{\Omega \backslash\{\omega\}}, p_{\omega}+\epsilon\right)$ with $\epsilon>0$ and all $\omega \in\left(\Xi \backslash \Xi^{\prime}\right) \cup\left(\Xi^{\prime} \backslash \Xi\right)$.

Let $n=\left\lfloor\frac{2 M}{\epsilon}\right\rfloor+1$ and let $T=([-n, n] \cap \mathbb{Z})^{\Omega}$. Note that $\delta$ is $\epsilon$-regular if $\delta+\epsilon T \subseteq \mathfrak{S}_{f}$. For each $\mathfrak{t} \in T$, the set of vectors $\delta$ such that $\delta+\epsilon \mathfrak{t} \in \mathfrak{S}_{f}$ is open and dense in $(-\epsilon, \epsilon)^{\Omega}$ since $\mathfrak{S}_{f}$ is open and dense in $\mathbb{R}^{\Omega_{f}}$. As $T$ is finite, it follows that the set of $\epsilon$-regular vectors contains an open and dense subset of $(-\epsilon, \epsilon)^{\Omega}$.

Q.E.D.

An arrangement $[\Xi ; p]$ is an $\epsilon$-equilibrium if every firm $f$ demands $\Xi_{f}$ when given access to all sales and to purchases in $\Xi$ at prices given by the price vector $p$, and to other purchases at prices given by the price vector $p+\epsilon$.

DEFINITION B.1: An arrangement $[\Xi ; p]$ is an $\epsilon$-equilibrium if $p \in[-2 M, 2 M]^{\Omega_{f}}$ and $\Xi_{f} \in D^{f}\left(\hat{p}^{f, \Xi, \epsilon}\right)$ for all $f$, where the price vectors $\hat{p}^{f, \Xi, \epsilon} \in \mathbb{R}^{\Omega_{f}}$ are defined by

$$
\hat{p}_{\omega}^{f, \Xi, \epsilon}= \begin{cases}p_{\omega} & \text { if } \omega \in \Xi_{f} \text { or } f=\mathrm{s}(\omega), \\ p_{\omega}+\epsilon & \text { if } \omega \notin \Xi_{f} \text { and } f=\mathrm{b}(\omega) .\end{cases}
$$


The following claim shows that $\epsilon$-equilibria exist.

CLAIM B.2: Under FS and BWP, there exists an $\epsilon$-equilibrium for each $\epsilon \in(0, M)$.

Proof: Let $\delta \in(-\epsilon, \epsilon)^{\Omega}$ be an $\epsilon$-regular vector, which exists by Claim B.1. Consider the sets $\mathfrak{P}_{\omega}=[-2 M, 2 M] \cap\left(\epsilon \mathbb{Z}+\delta_{\omega}\right)$ of prices, and let

$$
\widehat{X}=\bigcup_{\omega \in \Omega}\left(\{\omega\} \times \mathfrak{P}_{\omega}\right) \subseteq X .
$$

Note that $C^{f}$ is single-valued on $\mathcal{P}\left(\widehat{X}_{f}\right)$ due to the $\epsilon$-regularity of $\delta$, and hence we can write $C^{f}(Y)=\left\{\mathrm{C}^{f}(Y)\right\}$ for $Y \subseteq \widehat{X}_{f}$.

Following Hatfield and Kominers (2012), we define $\Phi: \mathcal{P}(\widehat{X})^{2} \rightarrow \mathcal{P}(\widehat{X})^{2}$ by

$$
\begin{aligned}
\Phi\left(X^{B}, X^{S}\right) & =\left(\Phi^{B}\left(X^{B}, X^{S}\right), \Phi^{S}\left(X^{B}, X^{S}\right)\right), \\
\Phi^{B}\left(X^{B}, X^{S}\right) & =\left(\widehat{X} \backslash X^{S}\right) \cup \bigcup_{f \in F} C^{f}\left(X_{\rightarrow f}^{B} \cup X_{f \rightarrow}^{S}\right)_{f \rightarrow}, \\
\Phi^{S}\left(X^{B}, X^{S}\right) & =\left(\widehat{X} \backslash X^{B}\right) \cup \bigcup_{f \in F} C^{f}\left(X_{\rightarrow f}^{B} \cup X_{f \rightarrow}^{S}\right)_{\rightarrow f} .
\end{aligned}
$$

As in Fleiner (2003), Hatfield and Milgrom (2005), Hatfield and Kominers (2012), and Fleiner et al. (2018), we order $\mathcal{P}(\widehat{X})^{2}$ by letting $\left(X^{B}, X^{S}\right) \sqsubseteq\left(\bar{X}^{B}, \bar{X}^{S}\right)$ if $X^{B} \supseteq \bar{X}^{B}$ and $X^{S} \subseteq \bar{X}^{S}$. As Hatfield and Kominers (2012) and Fleiner et al. (2018) showed, $\Phi$ is isotone (with respect to $\sqsubseteq$ ) under FS. The Tarski (1955) Fixed-Point Theorem guarantees that $\Phi$ has a fixed point $\left(X^{B}, X^{S}\right)$.

Given $f \in F$, since $\left(X^{B}, X^{S}\right)$ is a fixed point of $\Phi$, we have that

$$
X_{f \rightarrow}^{B}=\left(\widehat{X}_{f \rightarrow \backslash} \backslash X_{f \rightarrow}^{S}\right) \cup \mathrm{C}^{f}\left(X_{\rightarrow f}^{B} \cup X_{f \rightarrow}^{S}\right)_{f \rightarrow} .
$$

Since $\mathrm{C}^{f}\left(X_{\rightarrow f}^{B} \cup X_{f \rightarrow}^{S}\right)_{f \rightarrow} \subseteq X_{f \rightarrow}^{S}$, it follows that $X_{f \rightarrow}^{B} \cup X_{f \rightarrow}^{S}=\widehat{X}_{f \rightarrow}$. Taking unions over $f$, we have that

$$
\widehat{X}=\bigcup_{f \in F} \widehat{X}_{f \rightarrow}=\bigcup_{f \in F}\left(X_{f \rightarrow}^{B} \cup X_{f \rightarrow}^{S}\right)=X^{B} \cup X^{S} .
$$

Equation (B.3) also implies that

$$
X_{f \rightarrow}^{B} \cap X_{f \rightarrow}^{S}=\mathrm{C}^{f}\left(X_{\rightarrow f}^{B} \cup X_{f \rightarrow}^{S}\right)_{f \rightarrow} .
$$

Similarly, we have that $X_{\rightarrow f}^{B} \cap X_{\rightarrow f}^{S}=\mathrm{C}^{f}\left(X_{\rightarrow f}^{B} \cup X_{f \rightarrow}^{S}\right)_{\rightarrow f}$, and it follows that

$$
\left(X^{B} \cap X^{S}\right)_{f}=\mathrm{C}^{f}\left(X_{\rightarrow f}^{B} \cup X_{f \rightarrow}^{S}\right) .
$$

Let $\omega \in \Omega$ be arbitrary. Since $\epsilon<M$, we have that $\max \mathfrak{P}_{\omega}>M$ and $\min \mathfrak{P}_{\omega}<-M$. Hence, we have that $\left(\omega, \max \mathfrak{P}_{\omega}\right),\left(\omega, \min \mathfrak{P}_{\omega}\right) \notin X^{B} \cap X^{S}$ due to BWP and (B.5). If $\left(\omega, \max \mathfrak{P}_{\omega}\right) \notin X^{B}$, then adding $\left(\omega, \max _{\omega}\right)$ to $X^{B}$ and removing it from $X^{S}$ preserves (B.4) and (B.5) by BWP for $f=\mathrm{s}(\omega)$. Hence, we can assume that $\left(\omega, \max \mathfrak{P}_{\omega}\right) \in X^{B} \backslash X^{S}$. Similarly, we can assume that $\left(\omega, \min \mathfrak{P}_{\omega}\right) \in X^{S} \backslash X^{B}$. 
As $\mathfrak{P}_{\omega}$ is finite and $\left(\omega, \min \mathfrak{P}_{\omega}\right) \in X^{S}$, the set $\left\{p_{\omega}^{\prime} \in \mathfrak{P}_{\omega} \mid\left(\omega, p_{\omega}^{\prime}\right) \in X^{S}\right\}$ is finite and non-empty. Hence, we can define a price $p_{\omega}$ to be

$$
p_{\omega}=\min \left\{p_{\omega}^{\prime} \in \mathfrak{P}_{\omega} \mid\left(\omega, p_{\omega}^{\prime}\right) \in X^{S}\right\} .
$$

We claim that $[\Xi ; p]$ is an $\epsilon$-equilibrium, where $\Xi=\tau\left(X^{B} \cap X^{S}\right)$. As $\left(\omega, \max \mathfrak{P}_{\omega}\right) \notin X^{S}$

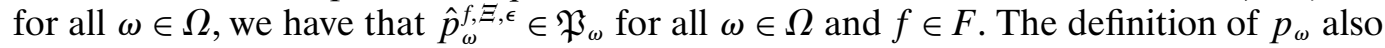
ensures that

$$
\left(\omega, \hat{p}_{\omega}^{\mathrm{b}(\omega), \Xi, \epsilon}\right) \in X^{B} \quad \text { and } \quad\left(\omega, \hat{p}_{\omega}^{\mathrm{s}(\omega), \Xi, \epsilon}\right)=\left(\omega, p_{\omega}\right) \in X^{S}
$$

for all $\omega \in \Omega$. It follows that

$$
\left(X^{B} \cap X^{S}\right)_{f} \subseteq \kappa\left(\left[\Omega_{f} ; \hat{p}^{f, \Xi, \epsilon}\right]\right) \subseteq X_{\rightarrow f}^{B} \cup X_{f \rightarrow}^{S}
$$

for all $f \in F$. Hence, (B.5) implies that $\Xi_{f} \in D^{f}\left(\hat{p}^{f, \Xi, \epsilon}\right)$ for all $f \in F$, so $[\Xi ; p]$ is an $\epsilon$-equilibrium.

Q.E.D.

As the interval $[-2 M, 2 M]$ is sequentially compact, Claim B. 2 implies that there exists an arrangement $[\Xi ; p]$, a sequence $n_{1}<n_{2}<\cdots$ of positive integers, and a sequence $p_{1}, p_{2}, \ldots \in[-2 M, 2 M]^{\Omega}$ such that $\left[\Xi ; p_{k}\right]$ is a $\frac{1}{n_{k}}$-equilibrium for all $k$ and $p_{k} \rightarrow p$. Note that $\hat{p}_{k}^{f, \nexists, \frac{1}{n_{k}}} \rightarrow p_{\Omega_{f}}$ for all $f \in F$ because $\frac{1}{n_{k}} \rightarrow 0$. Because $\Xi_{f} \in D^{f}\left(\hat{p}_{k}^{f, \exists, \frac{1}{n_{k}}}\right)$ for all $k$ and $D^{f}$ is upper hemicontinuous, it follows that $\Xi_{f} \in D^{f}\left(p_{\Omega_{f}}\right)$ for all $f \in F$. Thus, [ $\boldsymbol{\Xi} ; p$ ] is a competitive equilibrium.

\section{B.4. Completion of the Proof of Theorem 1}

Theorem B.1 and Lemmata B.1 and B.2 imply that the modified economy has a competitive equilibrium $[\Xi ; p]$, which must be a competitive equilibrium in the original economy by Lemma B.4.

\section{APPENDIX C: Other ProOfs OMitTed From the TeXT}

\section{C.1. Proof of Theorem 2}

Competitive equilibrium outcomes are clearly individually rational. It remains to show that no trail locally blocks a competitive equilibrium outcome. Let $[\Xi ; p]$ be a competitive equilibrium and let $A=\kappa([\Xi ; p])$. Suppose for the sake of deriving a contradiction that there is a locally blocking trail $\left(z_{1}, \ldots, z_{n}\right)$.

Let $z_{i}=\left(\omega_{i}, p_{i}^{\prime}\right)$. Let $f_{i}=\mathrm{s}\left(z_{i}\right)$ and let $f_{n+1}=\mathrm{b}\left(z_{n}\right)$. As $z_{1} \in Y$ for all $Y \in C^{f_{1}}\left(A_{f_{1}} \cup\left\{z_{1}\right\}\right)$ and $[\Xi ; p]$ is a competitive equilibrium, we must have that $p_{1}^{\prime}>p_{\omega_{1}}$. Similarly, as $z_{2} \in Y$ for all $Y \in C^{f_{2}}\left(A_{f_{2}} \cup\left\{z_{1}, z_{2}\right\}\right)$, we must have that $p_{2}^{\prime}>p_{\omega_{2}}$. A simple inductive argument shows that $p_{n}^{\prime}>p_{\omega_{n}}$. But we have that $p_{n}^{\prime}<p_{\omega_{n}}$ as $z_{n} \in Y$ for all $Y \in C^{f_{n+1}}\left(A_{f_{n+1}} \cup\left\{z_{n}\right\}\right)-$ a contradiction. Hence, there cannot be any locally blocking trails.

\section{C.2. Proof of Theorem 3}

We follow the proof strategy described in Section 5.4. We begin by defining locally semiblocking trails following Fleiner et al. (2018). 
Definition C.1: A trail $\left(z_{1}, \ldots, z_{n}\right) \in(X \backslash A)^{n}$ locally semi-blocks an outcome $A$ if:

- $z_{1} \in Y$ for all $Y \in C^{f_{1}}\left(A_{f_{1}} \cup\left\{z_{1}\right\}\right)$, where $f_{1}=\mathrm{s}\left(z_{1}\right)$; and

- for $1 \leq i \leq n-1$, we have that $\left\{z_{i}, z_{i+1}\right\} \subseteq Y$ for all $Y \in C^{f_{i+1}}\left(A_{f_{i+1}} \cup\left\{z_{i}, z_{i+1}\right\}\right)$, where $f_{i+1}=\mathrm{b}\left(z_{i}\right)=\mathrm{s}\left(z_{i+1}\right)$.

Let $A$ be an outcome, let $\Xi=\tau(A)$, and let $M$ be as in BWP. We define a set ${ }^{32}$

$$
X^{B}=\left\{z \in X \mid \text { there exists a locally semi-blocking trail }\left(z_{1}, \ldots, z_{n}\right) \text { with } z_{n}=z\right\} .
$$

For $\omega \in \Omega$, we define

$$
p_{\omega}=\min \left\{M, \inf _{\left(\omega, p_{\omega}^{\prime}\right) \in X^{B}} p_{\omega}^{\prime}\right\},
$$

so $p_{\omega}$ is the minimum of $M$ and the highest price at which $\omega$ is weakly undesirable to its seller. We prove that $\kappa([\Xi ; p])=A$ and that $[\Xi ; p]$ is a competitive equilibrium.

CLAIM C.1: Under BWP, if $A$ is individually rational, then we have that $\kappa([\Xi ; p])=A$.

Proof: Suppose that $\left(\omega, p_{\omega}^{\prime}\right) \in A$. BWP implies that $p_{\omega}^{\prime}<M$. As $u^{\mathrm{s}(\omega)}$ is strictly increasing in transfers and $A$ is individually rational, we have that $\left(\omega, p_{\omega}^{\prime \prime}\right) \in X^{B}$ if and only if $p_{\omega}^{\prime \prime}>p_{\omega}^{\prime}$. Hence, we have that $p_{\omega}=p_{\omega}^{\prime}$. As $\tau(A)=\Xi$, the claim follows.

Q.E.D.

CLAIM C.2: Under FS and BWP, if A is trail-stable, then $[\Xi ; p]$ is a competitive equilibrium.

Proof: Suppose for the sake of deriving a contradiction that $\Xi_{f} \notin D^{f}\left(p_{\Omega_{f}}\right)$. As $A$ is individually rational, it follows from Claim C.1 that $\Xi^{\prime} \notin D^{f}\left(p_{\Omega_{f}}\right)$ for all $\Xi^{\prime} \subseteq \Xi_{f}$.

We first perturb prices to ensure that sellers have strict incentives to propose contracts. Due to the upper hemicontinuity of demand, we can ensure that sufficiently small perturbations of prices do not affect the property that $f$ demands no subset of $\Xi_{f}$. Formally, we define a set $\mathfrak{O}$ of price vectors by

$$
\mathfrak{O}=\left\{p^{\prime} \in \mathbb{R}^{\Omega_{f}} \mid D^{f}\left(p^{\prime}\right) \cap \mathcal{P}\left(\Xi_{f}\right)=\emptyset\right\} .
$$

As $D^{f}$ is upper hemicontinuous (by Berge's Maximum Theorem) and $\mathcal{P}\left(X_{f}\right)$ is discrete, $\mathfrak{O}$ must be an open set. Hence, $\mathfrak{O}$ must contain an open ball of radius $(|\Omega|+1) \epsilon$ around $p_{\Omega_{f}}$

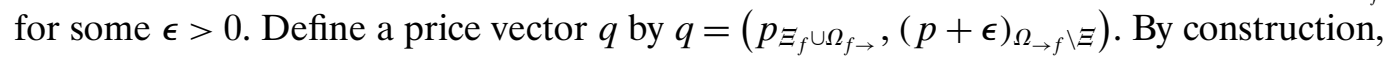
we have that $q \in \mathfrak{O}$, so $D^{f}(q) \cap \mathcal{P}\left(\Xi_{f}\right)=\emptyset$. Equation (C.1) ensures that $\left(\omega, q_{\omega}\right) \in X^{B}$ whenever $\omega \in \Omega_{\rightarrow f} \backslash \Xi$ is such that $p_{\omega}<M$.

By Theorem A.1, FSII must be satisfied. To produce a contradiction, we consider the set of trades that $f$ could demand at price vector $q$ that contains fewest trades outside $\Xi_{f}$. Formally, let $\Psi \in D^{f}(q)$ minimize $\left|\Psi^{\prime} \backslash \Xi\right|$ over all $\Psi^{\prime} \in D^{f}(q)$. Consider the corresponding set of contracts $W=\kappa([\Psi ; q])$. Note that $W \nsubseteq A$ and $W_{\rightarrow f} \backslash A \subseteq X^{B}$ by construction and BWP. Because $\Psi \in D^{f}(q)$, we have that $W \in C^{f}(W)$. Therefore, if $Z \in C^{f}(W)$, then we must have that $U^{f}(Z)=U^{f}(W)$ and hence that $\tau(Z) \in D^{f}(q)$. As a result, the choice of $\Psi$ ensures that

$$
Z \backslash A=W \backslash A \text { for all } Z \in C^{f}(W)
$$

\footnotetext{
${ }^{32}$ In the fixed-point interpretation of trail-stable outcomes (Fleiner et al. (2018), Adachi (2017)), $X^{B}$ is the set of contracts that are available to their buyers.
} 
We divide into cases based on whether $f$ is the seller of any contracts in $W \backslash A$ to produce a contradiction.

Case 1: $(W \backslash A)_{f \rightarrow} \neq \emptyset$. In this case, we either produce a locally blocking trail or show that each sale in $W \backslash A$ must appear in some locally semi-blocking trail. Formally, let $z \in W_{f \rightarrow} \backslash A_{f \rightarrow}$ be arbitrary. Equation (C.2) implies that $z \in Z$ for all $Z \in C^{f}(W)$. By IFSS, it follows that $z \in W^{\prime}$ for all $W^{\prime} \in C^{f}\left(A \cup\{z\} \cup W_{\rightarrow f}\right)$. Let $W^{0} \in C^{f}\left(A \cup\{z\} \cup W_{\rightarrow f}\right)$ minimize $\left|W^{\prime} \backslash A\right|$ over all $W^{\prime} \in C^{f}\left(A \cup\{z\} \cup W_{\rightarrow f}\right)$.

Let $\{\omega\}=\tau(\{z\})$. As $q_{\omega}=p_{\omega}$, the trail $\left(\left(\omega, p_{\omega}^{\prime}\right)\right)$ cannot be locally semi-blocking for any $p_{\omega}^{\prime}<q_{\omega}$ by (C.1). Hence, we must have that $A_{f} \in C^{f}\left(A_{f} \cup\{z\}\right)$ due to the continuity of $u^{s(\omega)}$ in transfers. It follows that $W_{\rightarrow f}^{0} \backslash A_{\rightarrow f} \neq \emptyset$. Since $W_{\rightarrow f} \backslash A \subseteq X^{B}$, there must exist a locally semi-blocking trail $\left(z_{1}, \ldots, z_{n}\right)$ with $z_{n} \in W_{\rightarrow f}^{0}$. By DFSP, we have that $z_{n} \in W^{\prime}$ for all $W^{\prime} \in C^{f}\left(A_{f} \cup\left\{z_{n}, z\right\}\right)$. We further divide into cases based on whether there exists $W^{\prime \prime} \in C^{f}\left(A_{f} \cup\left\{z_{n}, z\right\}\right)$ with $z \notin W^{\prime \prime}$ to derive contradictions.

Subcase 1.1: There exists $W^{\prime \prime} \in C^{f}\left(A_{f} \cup\left\{z_{n}, z\right\}\right)$ with $z \notin W^{\prime \prime}$. Then, the trail $\left(z_{1}, \ldots, z_{n}\right)$ is locally blocking, contradicting the hypothesis that $A$ is trail-stable.

Subcase 1.2: $z \in W^{\prime \prime}$ for all $W^{\prime \prime} \in C^{f}\left(A_{f} \cup\left\{z_{n}, z\right\}\right)$. Then, $\left(z_{1}, \ldots, z_{n}, z\right)$ is a locally semi-blocking trail. Since $u^{f}$ is continuous in transfers, there exists $p_{\omega}^{\prime}<p_{\omega}$ such that $\left(z_{1}, \ldots, z_{n},\left(\omega, p_{\omega}^{\prime}\right)\right)$ is a locally semi-blocking trail—contradicting the definition of the price vector $p$ from (C.1).

Case 2: $(W \backslash A)_{f \rightarrow}=\emptyset$. Let $z \in W \backslash A$ be arbitrary, and let $\left(z_{1}, \ldots, z_{n}\right)$ be a locally semi-blocking trail with $z_{n}=z$. Equation (C.2) implies that $z \in Z$ for all $Z \in C^{f}(W)$. By DFSP, it follows that $z \in Z$ for all $Z \in C^{f}\left(A_{f} \cup\{z\}\right)$. Thus, $\left(z_{1}, \ldots, z_{n}\right)$ is a locally blocking trail, contradicting the hypothesis that $A$ is trail-stable.

The cases exhaust all possibilities. We have produced contradictions in all cases, completing the proof of the claim.

Q.E.D.

Claims C.1 and C.2 together imply the theorem.

\section{C.3. Proof of Corollary 2}

Competitive equilibrium outcomes exist by Theorem B.1 and are trail-stable by Theorem 2. Trail-stable outcomes lift to competitive equilibria by Theorem 3 .

\section{REFERENCES}

ADACHI, H. (2017): "Stable Matchings and Fixed Points in Trading Networks: A Note," Economics Letters, 156, 65-67. [1635,1659]

AumAnN, R. J. (1964): "Markets With a Continuum of Traders," Econometrica, 32 (1-2), 39-50. [1634]

BALDWIN, E., AND P. KLEMPERER (2019): “Understanding Preferences: 'Demand Types,' and the Existence of Equilibrium With Indivisibilities," Econometrica 87 (3), 867-932. [1634,1636]

BLAIR, C. (1988): “The Lattice Structure of the Set of Stable Matchings With Multiple Partners," Mathematics of Operations Research, 13 (4), 619-628. [1646]

Cherchye, L., T. Demuynck, B. De Rock, And F. Vermeulen (2017): "Household Consumption When the Marriage Is Stable," American Economic Review, 107 (6), 1507-1534. [1635]

CHIAPPORI, P.-A. (1988): "Rational Household Labor Supply,” Econometrica, 56 (1), 63-90. [1639]

CRAWFORD, V. P., AND E. M. KNOER (1981): “Job Matching With Heterogeneous Firms and Workers," Econometrica, 49 (2), 437-450. [1634,1643] 
Danilov, V., G. Koshevoy, AND K. Murota (2001): "Discrete Convexity and Equilibria in Economies With Indivisible Goods and Money," Mathematical Social Sciences, 41 (3), 251-273. [1636]

Dupuy, A., A. Galichon, S. JAFFe, And S. D. Kominers (2017): “Taxation in Matching Markets,” available at SSRN, https://papers.ssrn.com/sol3/papers.cfm?abstract id=3060746. [1636,1639]

FleINER, T. (2003): "A Fixed-Point Approach to Stable Matchings and Some Applications," Mathematics of Operations Research, 28 (1), 103-126. [1657]

Fleiner, T., R. JAgAdeESAN, Z. JANKó, AND A. Teytelboym (2019): "Supplement to 'Trading Networks With Frictions'," Econometrica Supplemental Material, 87, https://doi.org/10.3982/ECTA14159. [1636]

Fleiner, T., Z. JANkó, A. TAmurA, And A. Teytelboym (2018): “Trading Networks With Bilateral Contracts," available at SSRN, https://papers.ssrn.com/sol3/papers.cfm?abstract_id=2457092. [1635,1643,1645, $1646,1648,1649,1656-1659]$

FOX, J. T. (2017): "Specifying a Structural Matching Game of Trading Networks With Transferable Utility," American Economic Review, 107 (6), 256-260. [1635]

(2018): "Estimating Matching Games With Transfers," Quantitative Economics, 9 (1), 1-38. [1635]

FoX, J. T., D. H. HsU, AND C. YANG (2018): "Unobserved Heterogeneity in Matching Games With an Application to Venture Capital," Journal of Political Economy, 126 (4), 1339-1373. [1635]

GALE, D., AND L. S. SHAPley (1962): "College Admissions and the Stability of Marriage," American Mathematical Monthly, 69 (1), 9-15. [1634,1635,1645]

Galichon, A., S. D. Kominers, AND S. WeBER (2019): “Costly Concessions: An Empirical Framework for Matching With Imperfectly Transferable Utility,” Journal of Political Economy (forthcoming). [1635,1636, 1639]

Gul, F., AND E. STACCHETTI (1999): “Walrasian Equilibrium With Gross Substitutes,” Journal of Economic Theory, 87 (1), 95-124. [1636,1641]

HATFIELD, J. W., AND S. D. KOMINERS (2012): "Matching in Networks With Bilateral Contracts," American Economic Journal: Microeconomics, 4 (1), 176-208. [1634,1635,1643,1644,1650-1652,1656,1657]

Hatfield, J. W., AND P. Milgrom (2005): "Matching With Contracts," American Economic Review, 95 (4), 913-935. [1634,1644,1657]

HATField, J. W., R. JAGAdEesan, AND S. D. Kominers (2019): "Matching in Networks With Bilateral Contracts: Corrigendum,” American Economic Journal: Microeconomics (forthcoming). [1642,1652]

Hatfield, J. W., S. D. Kominers, A. Nichifor, M. Ostrovsky, AND A. WestKamp (2013): "Stability and Competitive Equilibrium in Trading Networks," Journal of Political Economy, 121 (5), 966-1005. [1634-1638, 1641-1644,1646-1650,1652]

(2018): “Chain Stability in Trading Networks," Working Paper. [1634-1636,1649,1650]

(2019): "Full Substitutability," Theoretical Economics (forthcoming). [1634,1642,1651-1653]

Kelso, A. S., AND V. P. CRAWFORD (1982): "Job Matching, Coalition Formation, and Gross Substitutes," Econometrica, 50 (6), 1483-1504. [1634,1641,1643,1648]

Milgrom, P., AND C. SHAnnon (1994): “Monotone Comparative Statics,” Econometrica, 62 (1), $157-180$. [1652]

Ostrovsky, M. (2008): "Stability in Supply Chain Networks," American Economic Review, 98 (3), $897-923$. $[1634,1635,1641,1643,1646,1649,1650]$

PYCIA, M., AND M. B. YenMEZ (2017): “Matching With Externalities,” Working Paper. [1650]

RosteK, M., AND N. Yoder (2018): "Matching With Complementary Contracts," available at SSRN, https: //papers.ssrn.com/sol3/papers.cfm?abstract_id=3234080. [1650]

Roth, A. E. (1984): "Stability and Polarization of Interests in Job Matching," Econometrica, 52 (1), 47-58. $[1634,1644]$

SCHLEGEL, J. C. (2019): “Trading Networks With General Preferences,” available at SSRN, https://papers.ssrn. com/sol3/papers.cfm?abstract id=3230767. [1650]

Sun, N., AND Z. YANG (2006): "Equilibria and Indivisibilities: Gross Substitutes and Complements," Econometrica, 74 (5), 1385-1402. [1636]

TARSKI, A. (1955): “A Lattice-Theoretical Fixpoint Theorem and Its Applications," Pacific Journal of Mathematics, 5 (2), 285-309. [1657]

WestKamp, A. (2010): "Market Structure and Matching With Contracts," Journal of Economic Theory, 145 (5), 1724-1738. [1635]

Co-editor Joel Sobel handled this manuscript.

Manuscript received 8 February, 2016; final version accepted 15 April, 2019; available online 15 April, 2019. 